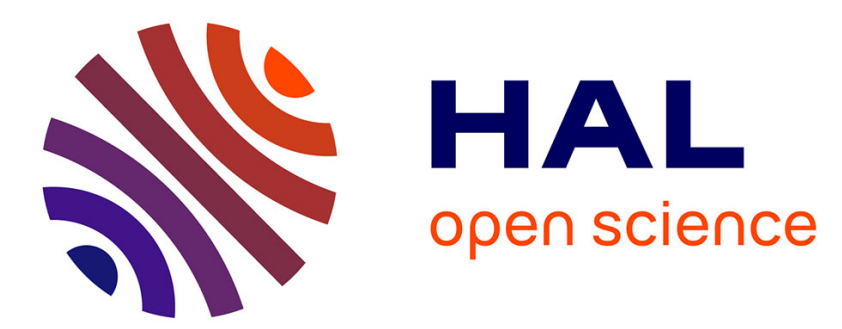

\title{
Mosaïques de la région de Vienne (Isère)
}

Henri Stern

\section{To cite this version:}

Henri Stern. Mosaïques de la région de Vienne (Isère). Gallia - Fouilles et monuments archéologiques en France métropolitaine, 1971, 29 (1), pp.123-149. 10.3406/galia.1971.2573 . hal-01934473

\section{HAL Id: hal-01934473 \\ https://hal.science/hal-01934473}

Submitted on 11 Mar 2020

HAL is a multi-disciplinary open access archive for the deposit and dissemination of scientific research documents, whether they are published or not. The documents may come from teaching and research institutions in France or abroad, or from public or private research centers.
L'archive ouverte pluridisciplinaire HAL, est destinée au dépôt et à la diffusion de documents scientifiques de niveau recherche, publiés ou non, émanant des établissements d'enseignement et de recherche français ou étrangers, des laboratoires publics ou privés.

\section{(이) $\$$}

Distributed under a Creative Commons Attribution - NonCommercial - NoDerivatives $\mid 4.0$ 


\title{
MOSAÏQUES DE LA RÉGION DE VIENNE (ISÈRE)
}

\author{
par Henri STERN
}

Je donne ci-apres les renseignements nouveaux que jai pu recueillir sur quatre mosaïques de pavement trouvées au siècle dernier aux environs de Vienne et qui portent

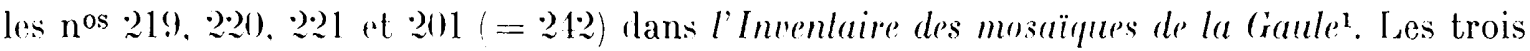
premières, considérées comme perlues. ont été retrouvées dans trois musées différents; pour la derniere, acquise il y a un siècle et demi par le Musée des Beaux Arts de Lyon et glétruite récemment par un incendie, nous pouvons présenter quelquess documents inédits.

\section{Las trots mosaigues Grange.}

Au début de l'année 1899, un cultivateur de Saint-Romain-en-Gal (Rhòne)2 du nom de Grange fou (iranges) al son fermier Prost, travaillant une vigne pres de la rive droite du Bhòne (en face de Vienne), avaient déterré plusieurs (3) ou 4) mosaïques33. Grange, le propriédaire du Lrrain. on avait proposé l'acquisition à divers musées (Lyon, Paris), mais sans succes. Ausis la trouvaille fut-elle recouverte et resta-t-elle sous terre penclant une douzaine

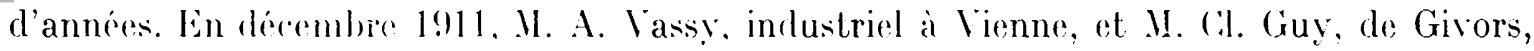
avant entendu parler de cette fouille déciderent de la reprendre. Ils en acheterent le droit au propriétaire et réussirent au cours de l'année 1912 a sortir de terre trois mosaïques $\left(n^{n s} 219 \text { a } 2 \cdot 21 \text { de l'Ine'enlaire }\right)^{4}$. Eiles furent transportées dans la propriété (sic) de MI. Vassy et ciuy qui tenterent de nourrau de les plarer auprese des musées de France, mais n'curent pas plus de succes que leur devancier. Par l'intermédiaire de l'architecte Rogatier Le Xail, de Lyon. ils les proposerent alors au British Museum. Le conservateur du département des antiquités greeques et romaines de ce Musée, Arthur Iamilton Smith,

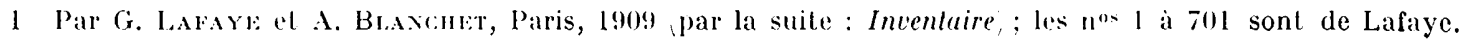

$\approx$ L; Incentaire indique par erreur Sainle-Colombe.

3 Voir E. Brzor, conservateur du Iluse de Vienne, dans Joturnal de Vienne el de Isere, ler avril 1899.

4 Les renseignements que je donne sur cetle decourerte sont tirés d'un article de A. VAssy et Cl. Ger, Mise a jour et description de trois mosäques romaines a saint-liomain-en-Gal (lhome) pres lienne lsere), dans

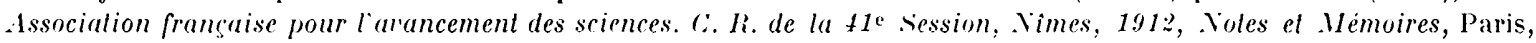
1913, p. 6:8-63:2. que ma signale Melle Iancha ef. infra, n. 9: al de trois journaux lyomnais : Le Progres, du 25

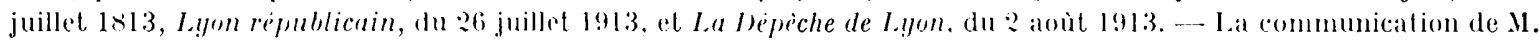

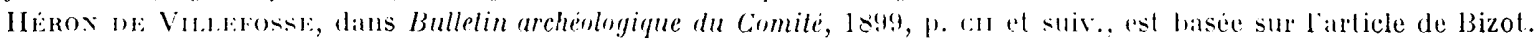

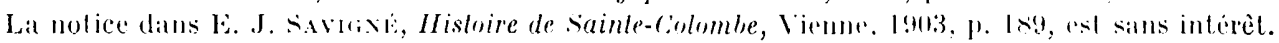


se rendit à Vienne et se fit acquéreur de l'une des trois pièces, le $n^{0} 220$, qu'il fit transporter à Londres par voie de mer, via Marscille5. Elle fut exposée dans ce Musée où elle se trouve touịours ${ }^{6}$.

La seconde mosaïque, Inv. no 219, Orphée parmi les animaux, vient d'ètre identifiée au Musée .J. Paul Getty à Malibu (Californie). La troisième, décorative, a tout de mème dû être acquise par le Musée de Vienne où elle se trouve à peu près intacte. La fouille de MM. Vassy et ciuy n'a done pas été en vain : les trois pavements qu'elle arait liv rés, existent toujours.

En 1902, le mème Grange reprit des sondages dans son terrain qui firent apparaitre quatre autres mosaïques dont Héron de Villefosse el L. Espérandieu rendirent compte aux Antiquaires de France. L'une d'elles (In'., no '2) 4), représentant Ilylas surpris par les Nymphes, fut achetée par le Général Beylié et donnée au Ilusée de Grenoble qui la conserve toujours. Le n ${ }^{0} 222,1$, deux perroquets perchés sur des vases, aurait été détruit pour réparer le $\mathrm{n}^{0} 224^{9}$; le $\mathrm{n}^{0} 222,2$ fut recouvert, le $\mathrm{n}^{0} 223$, partiellement dégagé (une tête de femme), pourrait bien se trouver au Musée de Vienne ${ }^{10}$. Yous ne nous arrèterons qu'aux mosaïques dégagées de nouveau en 1912 .

La découverte de 1902 portait au nombre de sept les pavements trouvés dans la propriété Grange. A en croire la notice de La Dépếche de Lyon ${ }^{11}$ ils appartenaient tous à la même habitation romaine. Iluit pièces, desservies par un couloir'11 bis ct pourvues d'un système de chauffage, furent déterminées et le plan (perdu aujourd'hui) fut levé par l'architecte Rambaud, conseiller municipal de Lyon.

La mosä̈que Inv. no 220 (au Brilish Museum). Iesurant au moment de la découverte $3,60 \mathrm{~m} \times 3,20 \mathrm{~m}$, elle a été réduite à $2,74 \mathrm{~m} \times 2,74 \mathrm{~m}$ par l'enlèvement des bandes de raccord blanches, dépourvues de décor (fig. 1). Quatre clichés au Musée de Vienne : une vue d'ensemble et trois détails, bien que mal éclairés et flous (fig. 1 à 4), permettent de juger de l'étal du pavement au moment de la découverte ${ }^{12}$. Selon E. Bizot ${ }^{13}$ il était assez bien conservé

5 La dépense notée dans l'inventaire du Musée, $2.10 \mathfrak{E}$, comprentil sans doute le prix d'achat, les frais de transport et de restauration.

6 Cif. R. P. IIxks, Calalogue of the Greek, Etruscan and Roman Painlings and .losaics in the Brilish Museum, Londres, 1933, p. 67 et suiv, nos 7a-e. L'auteur cite par erreur La Jepeiche de Lyon du ler au licu du 2 aoùt 1913.

7 Inventaire, nos $222,223,1$ et $2,224$.

8 Cr. Bulletin de la sociele nationale des Anliquaires de France, 1919, p. 133 el 15.1 al suiv; Iréron de Villefosse ne se souvenait plus à ce moment de la decouverte de $1 \times 99$, dont il avait rendu compte lui-mème lors d'une séance de la Commission des travaux hisloriques, l.e. et a laquelle Grange faisail allusion dans une leltre communiquée par IIéron de Villefosse.

9 Ithe J. Iancha, Assistante a la baculti des Iettres de Lyon, qui prepare sous ma direction une thise de 3e eycle sur les mosaïgues de Vienne, vient de retrourer les deux perroguets au Musée de Grenoble.

10 Plusieurs fragments de mosaique, représentant une tète de femme se trouvent an Musée lapidaire de liemne. IIte Lancha donnera des précisions à ce sujet.

11 L.c.

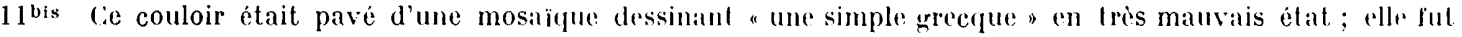
laissée en place (Vassy et GuY, o. l., p. 628).

12 Le Journal de Vienne el de l'Isire, l.c., mentionne les photographies d'un nommé Eymard; Héron dE Viltefosse, dans Bulletin arch. du Comite, l.c., dit avoir reçu, par les soins de Tony (haumartin, une photographie trop obscure. Le Progrès, l.c., donne la vue d'un photographe prenant un cliche "des mosaïques" en place. I.yon républicain, l.c., reproduit ta vue d'ensemble des archives du Ifusée de Vienne que nous pullions fig. 1 . Il y a de fortes chances qu'elle ait été prise, comme les trois autres, en 1912.

13 L.c. 


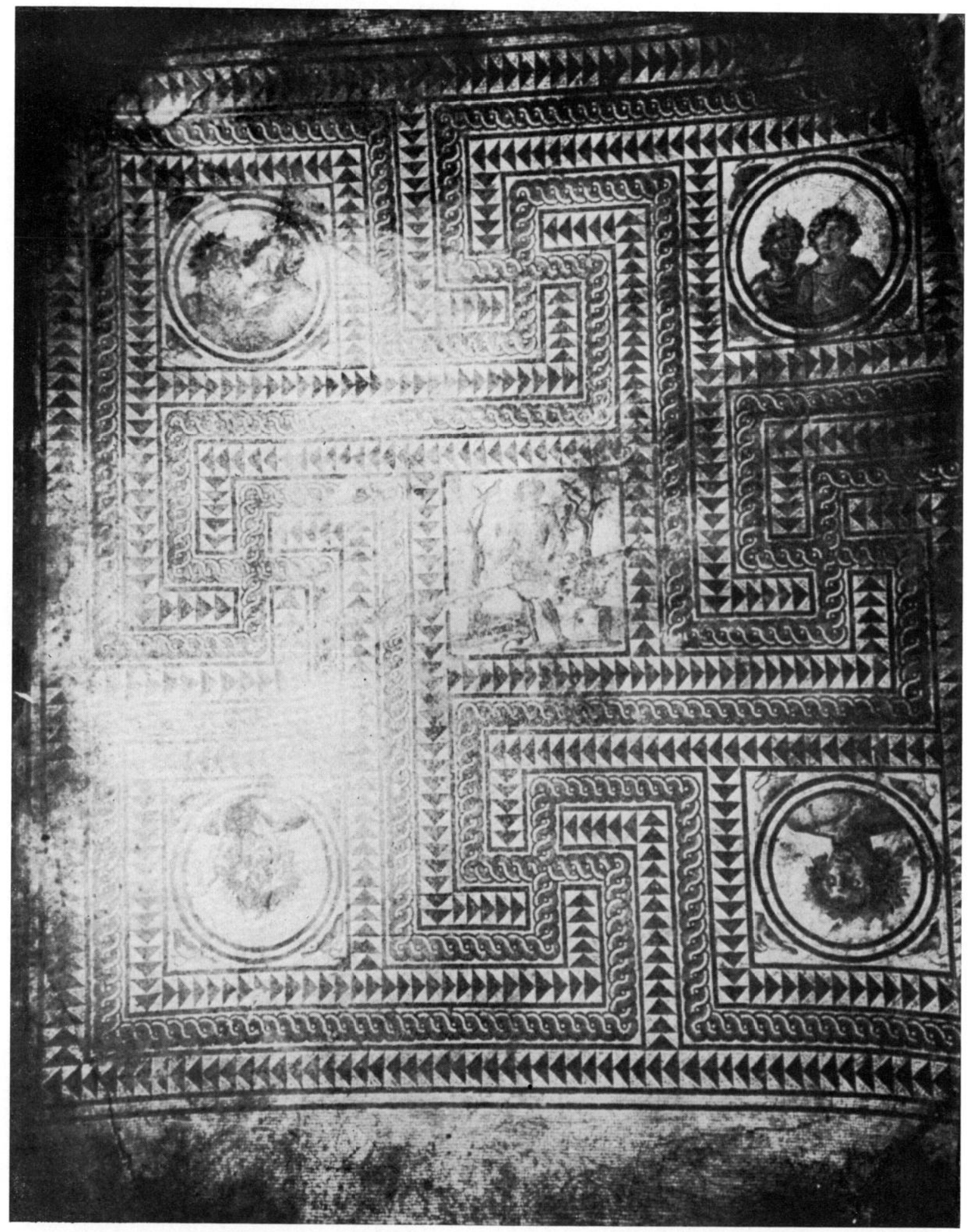

1 Mosaicgue Grange, Inv., no 220 , à sujet bachique, photographie prise probablement en 1912. 


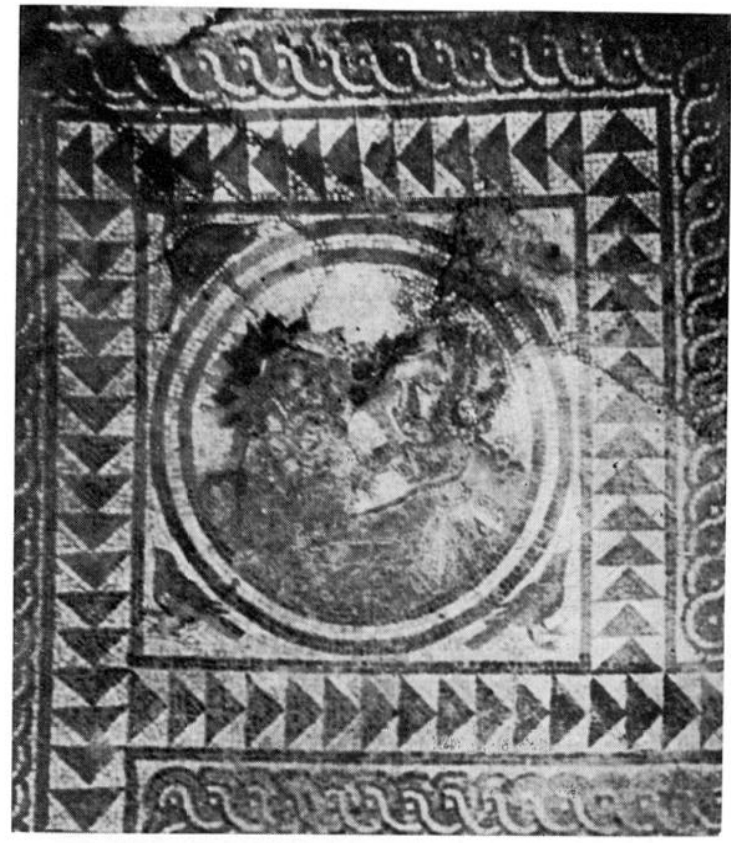

2 Couple bachique, de la même mosaiqque, phologralphie de 1912.

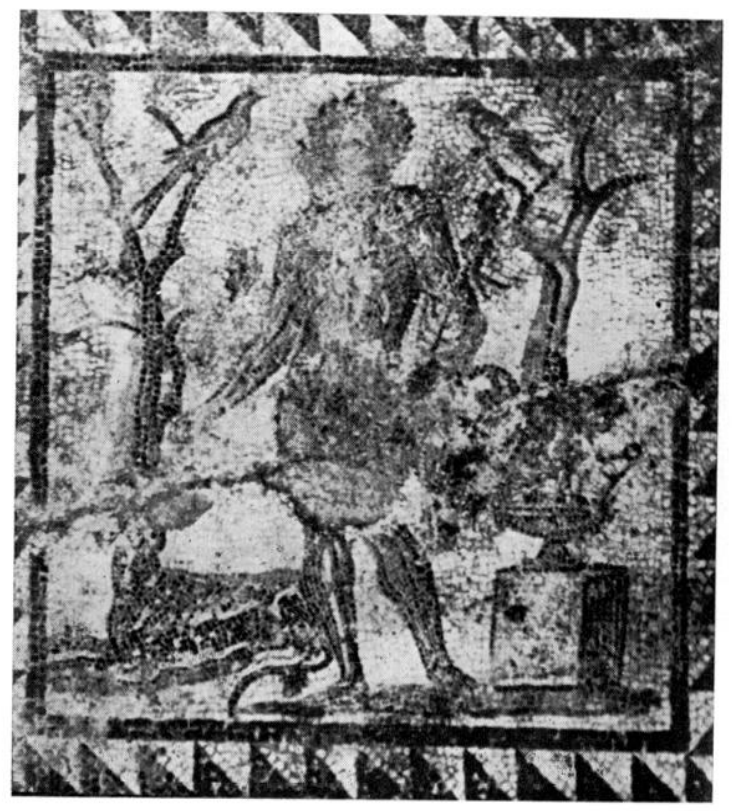

4 Dionysos, de la même mosaique, photographie de 1912.

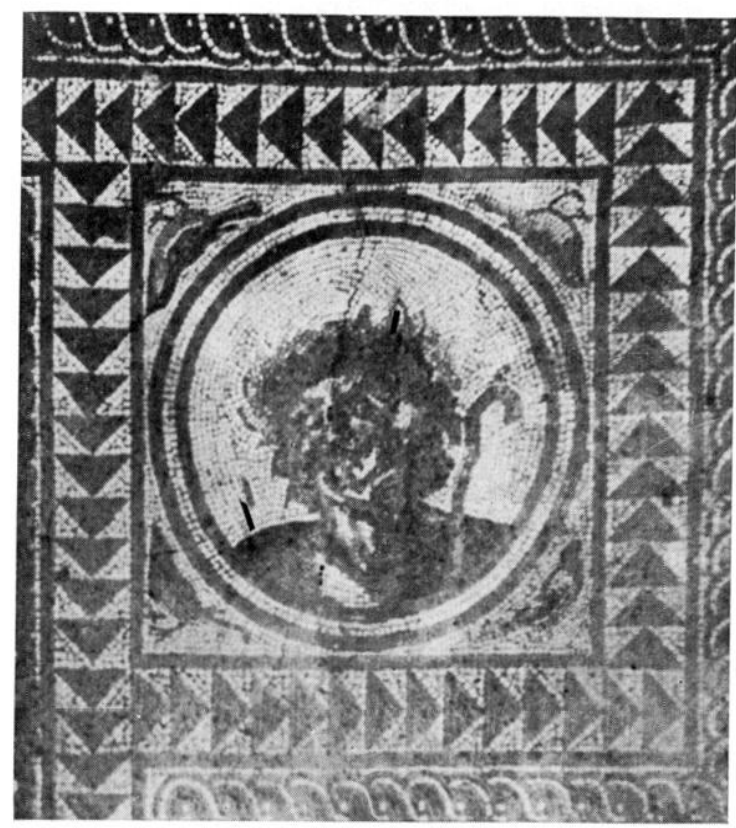

3 Satyre, de la même mosaïque, photographie de 1912 .

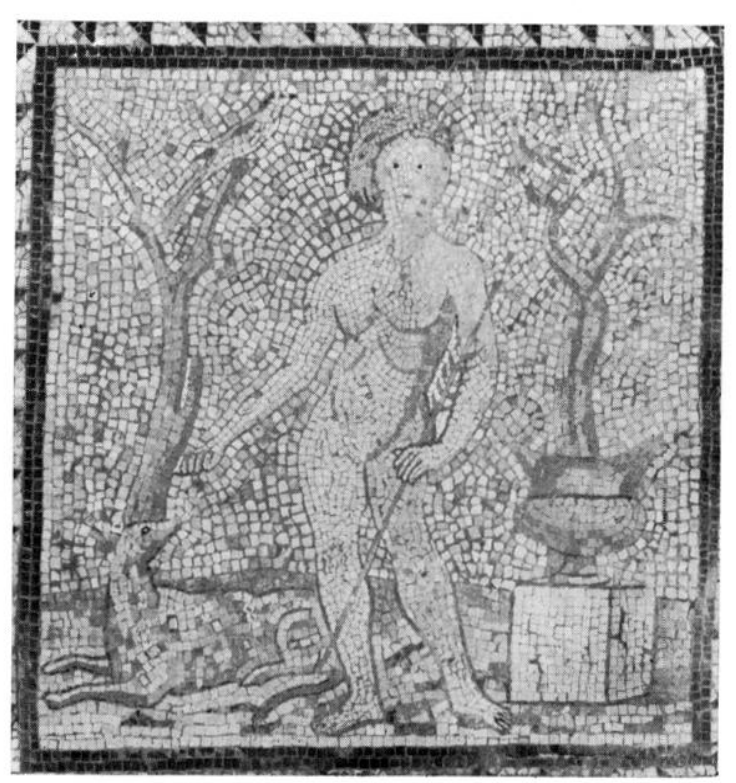

5) Dionỵsos, de la même mosaĩque, restauré, Musíc Britannique.

en 1899 : une mince brèche seulement traversait de part en part le tahleau central un peu au-dessous de l'axe horizontal (fig. 4). Si les photographies anciennes datent de 1912, ce que je suis porté à croire, la mosäque n'aurait pas souffert de son enterrement pendant douze ans; elle y est à peu près dans l'état décrit par Bizot. C"est d'autant plus probable, 
que les journaux de 1913 insistent sur son excellent état de conservation ${ }^{14}$. I'apres Lyon républicain le mosaïste Bertin de Lyon ne fut chargé que de la nettoyer. La réfection presque totale que l’on constate aujourd'hui aurait donc été nécessaire à cause des détériorations consécutives au transport de Vienne à Londres et lors de la mise en place au Musée Britannique ${ }^{15}$.

Le caneras n'a pas éti modifié. I'n entrelacs a deux brins, noir, blanc et rouge. avec du jaune. une bande à triangles-rectangles isocèles se touchant par la pointe, noirs sur fond blanc, forment quatre grands srastikas autour de quatre carrés dans les angles et d'un autre all milieu (fig. 1). Les bandes sont séparées par des filets noirs. Dans les carrés d'angle s'inscrivent des médaillons à sujets figurés, dans celui du centre un tableau à personnage courre toute la surface.

Les bustes dans les médaillons, pris par Bizot pour les representations des quatre saisons. ont ete correctement interprétés par Hinks ${ }^{16}$. En haut a gauche (fig. 2) on voit un couple hachique, un silene barbu et une jeune ménade, tous deux couronnés de lierre et drapés de rouge (le tableau est entièrement refait, fig. 6). Sur la photographie ancienne on distingue à peine derant l'homme un vase à fruits mentionne par Bizot. Il a été transforme par le restaurateur en un objet informe (fig. 6). -- En haut a droite un autre couple bachique (fig. 1), un silène cornu ${ }^{17}$, une tunique exomide rouge agrafée sur son épaule droite, se tient à còté d'une femme rètue d'une tunique bleu grisâtre et d'un manteau rouge. Le médaillon inférieur de droite inscril un beau buste de satyre nu (fig. 8), le mieux conservé de tous, à la chevelure abondante couronnée de lierre. Son regard languissant se dirige vers le personnage du médaillon en face (fig. 3), le buste d'un aulre satyre aux cheveux hirsutes qui tient un bâton de berger (pedum). Les quatre médaillons sont cantonnés d'oiseaux vus de profil. - Sauf la tète du jeune satyre et quelques oiseaux, tous les sujets ont été refaits dans un style sec et sans relief (fig. i) it 7,9$)$.

Le personnage du tableau central, vu de face (fig. 1), tourne la tète légèrement vers la grauche, le corps musclé est nu, sauf des pans de manteau sur l'épaule et l'avant hras gauche. Les traits étaient à peu près effacés. Vigoureusement modelé à l'orignine, ce corps est maintenant d'une teinte absolument plate comme les traits du visage (fig. 5). Les cheveux sont couronnés de feuillage. De la main gauche l'homme tient un objet que R. P. Hinks appelle une flèche, ourre évidente du restaurateur. ("était un thyrse à rubans (fig. 4), les stries autour de l'avant-bras, devenues les pennes de la llèche, indiquaient les plis d'une draperie. La partic basse des cuisses élait détériorée par la brèche. A la gauche de ce personnage un grand cratère à deux anses, complètement refait, est posé sur une base cubique. A sa droite un animal a longue queue est areroupi ; l'homme lui tend sa main droite refermée sur un objet incertain. Tous les observateurs ont considéré cet animal comme un chien qui tourne

14 Il faut ividemment tenir compte du fait que ces journaux, pour faire ressortir l'erreur des musees francais d'en avoir refuse lachat, abondent en eloge sur le remarquable elal du parement.

15) Aucun document ne renseigne sur ces operations.

16 L.e.

17 (es cornes, nellement visibles sur le cliche ancien el rouserveres frar lo restaurateur fig. 7 ), ne sont. mentionnes par ancun observateur. Bizot les considere comme ane coiffure en forme de demi-lume, semblable à celle d'Arlemis. 

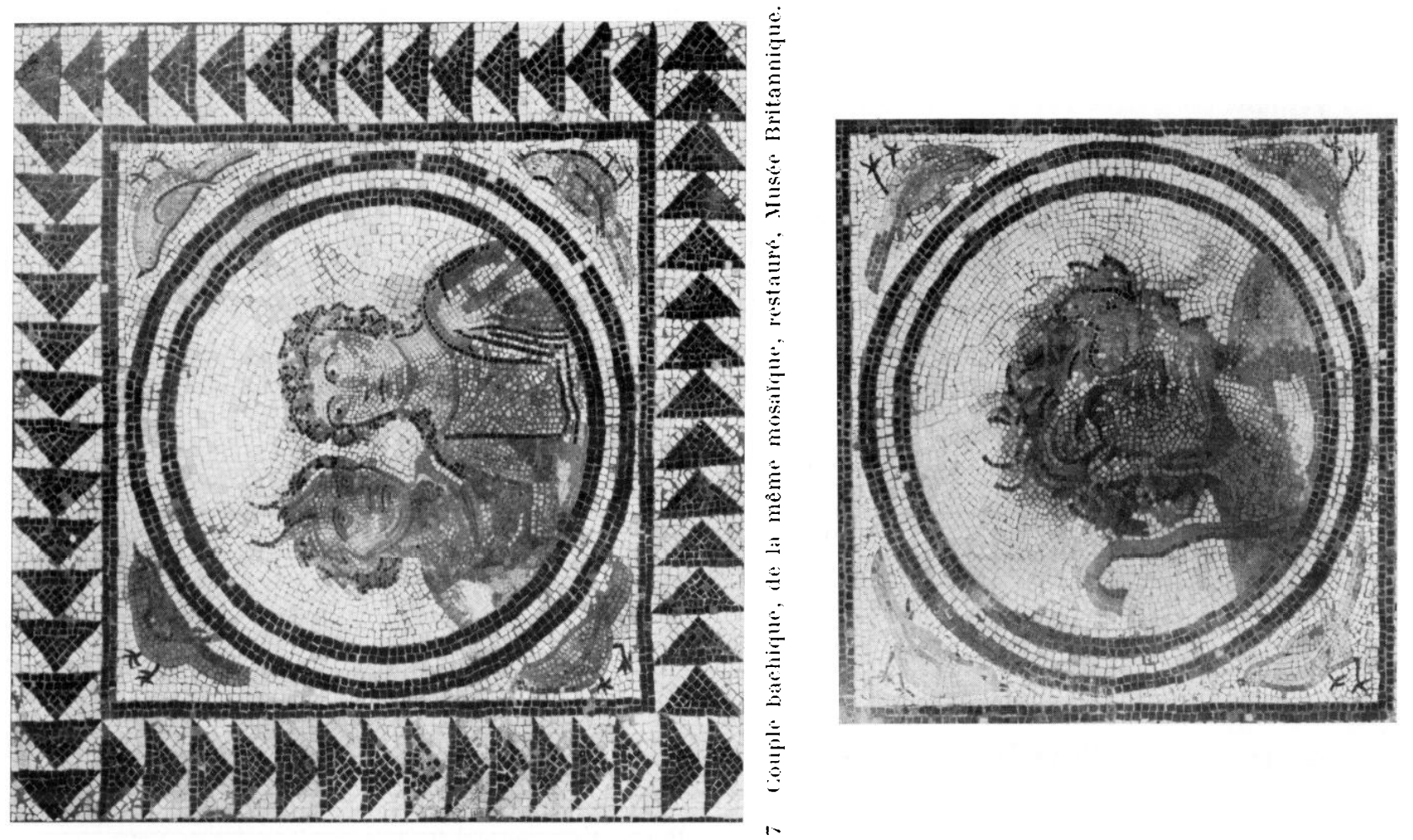

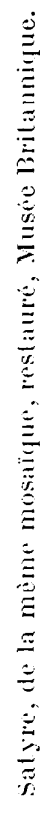
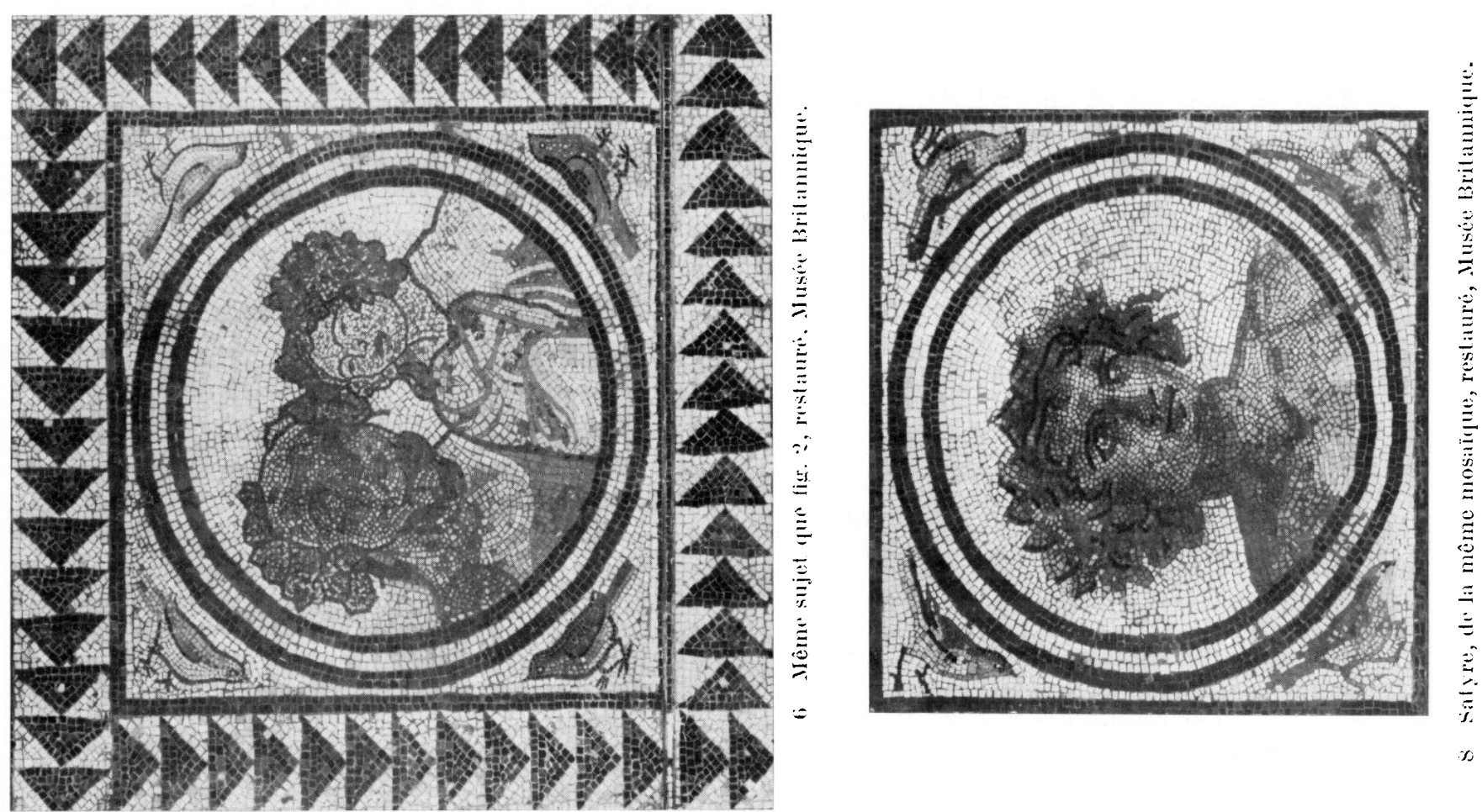
son museau pointu vers son maitre. Or, ce qui paraissait ètre le contour de sa tète, n'était "n réalité qu une lacune que le restaurateur a transformé en tête de chien. De part et d'autre de ce personnage se dresse un arbre dénudé avec un oiseau, tournant le bec vers la tète de l'homme. Tous les sujets ont été contournés de noir par le mosaïste moderne.

Le personnage du tableau eentral a été considéré tantôt comme un chasseur ${ }^{18}$, tantôt comme Silvain ${ }^{19}$. Bizot ${ }^{20}$ proposait d'y reconnaitre le propriétaire de la villa. Si l'on accepte notre description, ces commentaires sont à éarter, et il ne reste qu'une seule interprétation possible : il s'agit de Dionysos entouré du cortège bachique. Le thyrse et le cratère sont les attributs habituels du dieu. l'animal à ses pieds ne peut ètre qu'un tigre ou une panthère. Les deux oiseaux seulement ne cadrent pas aves son iconographie. Le restaurateur ayant morlifié les couleurs, l'identification en est malheureusement impossible aujourd'hui. I'après Bizot ils étaient du mème ton gris-noir que les arbres; d'après R. P. Hinks l'oiseau de gauche a une longue queue rouge. des ailes rouges et roses. le poitrail jaune, la tête et le bec noirs, celui de droite à queue plus courte, est semblable. Ies divinités mâles ou femelles de la Gaule peuvent être flanquées de deux oiseaux tournés vers leur tête ${ }^{21}$ qui, selon certains savants. seraient des oiseaux oraculaires ${ }^{22}$. Sans remplir cette fonction ici, ils pourraient bien ètre inspirés par une tradition iconographique gauloise.

Dionysos inscrit dans un ranevas géométrique. se retrouve deux fois sur des mosaïques de la région. In parement de Lyon ${ }^{23}$ le présente monté sur une panthère, entouré des bustes des quatre saisons, le tout inserit dans un fond de svastikas. Ln fragment, provenant de Vienne, qui se trouve aujourd hui dans un petit musée (une sorte de remise) à SaintGeorges-de-Rheneins: (Rhòne) (petite localité entre Mâcon et Lyon)24, a fait partie d'une mosaïque qui a dì ìtre comme le double de la nòtre. Ln seul panneau figuré en est conservé, le buste d'un satyre barbu, couronné de lierre. les cheveux enserrés par un ruban ; il est vêtu d'une pardalide, agrafée sur l'épaule droile. ch lient un pedum. Le panneau carré, non circulaire (qui a les dimensions de ceux de Sainte-Colombe) s̈inscrit sur un fond de svastikas formées d'un entrelacs à deux brins et d'une bande de triangles se touchant par la pointe. Le style est vigoureux, mais moins nuancé et moins fin que celui de la mosaïque Grange. Le canevas du fond, des svastikas qui renferment des petits panneaux, caractéristique du style des ateliers de Vienne et de Lyon, a souvent été étudié ces temps derniers et il est superflu d'y revenir.

Les oiseaux inscrits dans les écoinçons des médaillons, encore que rares, n'en sont pas moins typiques du style des ateliers rhorlaniens de la fin du II $^{\mathrm{e}}$ et de la première moitié

18 Lyon republicain, l.c.: Mepeche de Lyon, l.c.

19 R. P. IIxks, l.c.

?() L.c.

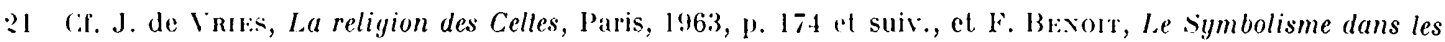
sancluaires de (jaule coll. Latomus, vol. 105), Bruxelles, 1970, p. 65 et suiv.

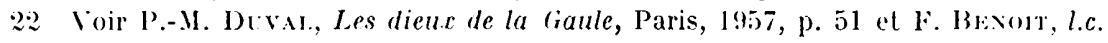

23 II. Srinx, Recueil genéral des mosaïques de la Gaule, Xe suppl. à Ciallia, II, no 18 !:- Recueil, 11 ).

24 II me 11 . Blanchard-Lemee, chargée de recherche au C.N.R.S., qui collabore a la priparation du t. II, 2 du Recueil géneral des mosaiques de la Giale, a bien voulu me signaler ce fragment.

25) $I$ me V. ron Gonzenbach, la premiere, a attire l'allention sur ce canevas rhod:mien dans Die römischen

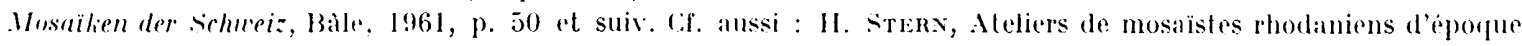
gallo-romaine, dans lat mosaique greco-romaine, Paris, l965, p. 2:38 el suiv, ; Recueil, 1, no:97 G; Recueil, II, nos 47 , 48, 76, 138. 


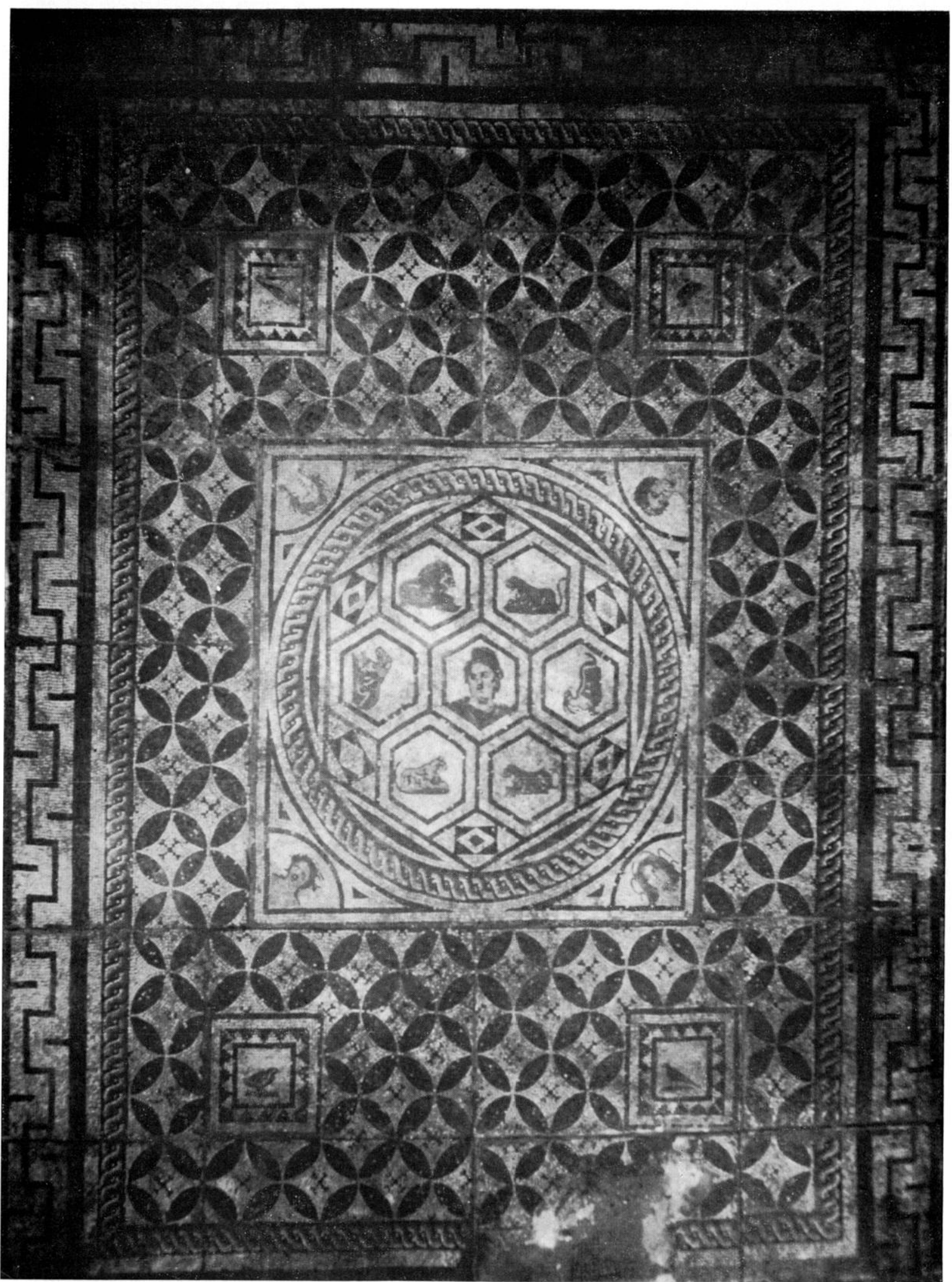

10 Lal mosaïlute d'orpher, apres de la decouverle en 191:. 
du II $^{\mathrm{e}}$ siècle. Des échantillons se trouvent dans la mosaïque de l'iuresse d'Hercule de Vienne ${ }^{26}$, sur les fragments de parements du .Iusée lapidaire de Vienne et sur des mosaïques découvertes récemment à Saint-Romain-en-(ral (tous inédits).

La deuxième des trois mosaüques déterrées par MII. Vassy et Guy est conservée aux États-Unis. M. Stothart, Assislant-Curalor du Musée J. Paul Getty à Malibu (Cal.), où elle se trouve, donne ci-dessous des précisions à son sujet ${ }^{27}$, auxquelles nous ajouterons quelques indications supplémentaires.

Lors de la construction du Musée J. Paul Gelly à Malibu, fut entreprise une installation inhabituelle et coùteuse, réclamant des soins méticuleux : il s'agissait de remonter un pavement de mosaïque romain à partir de plus de vingt panncaux (fig. 10, 11). Et, bien qu'on ait dépensé beaucoup de peine et de temps ì assembler et a poser le pavement, on nesarait qu'étonnamment peu de choses sur la mosaïque. I.e vendeur n'avait indiquée ${ }^{28}$ ni provenance exacte ni date précise ${ }^{29}$. Mais après qu'on l'ait soigneusement remontée et qu'on l'ail exposée dans le Musée, plusieurs savants ont donné d'eux-mèmes des avis aulorisés sur l'important problème de sa date et de sa provenance, ainsi que sur l'identification exacte de son sujet.

Franklin Biebel. dans une lellre au regretté W. R. Valentiner, le précédent directeur du Musée Getty, fail justement remarquer qu'une datation au v'e s., proposée par l'antiquaire Barsanti qui l'a reprise à l'inventaire de la collection Ilearst, est manifestement erronée, el que le ir ${ }^{\mathrm{e}}$ ou le III siecle sont plus vraisemblables. II suggò̀re en outre que le sujet est Orphée entouré des animaux et indique que l'on a trouvé un autre exemple d'un schéma identique «sur un pavement découvert

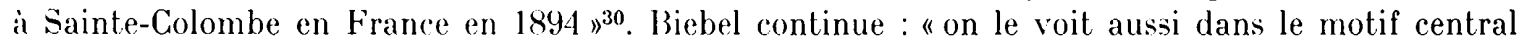
du pavement d'un bain romain fouillé en 1854 à proximité de la Porta Pia, ainsi que dans les Loges de Raphä̈l au Valican $»^{31}$.

La référence de Biebel à l'Invenlaire de Lafaye est, très précieuse. La notice qu'il cite (no 217) décril un pavement mis au jour à sainte-Colombe ${ }^{32}$, dont la composition est apparentée à celle du

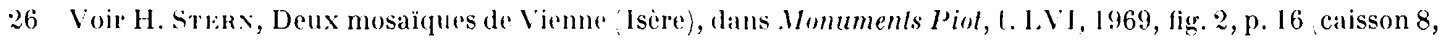
per(u), fig. 20, p. 33 ; caisson 24), fig. 2:3, p. 3.4 ; caisson :28:.

27 Texte aimablement traduit el communiqui par le Deparlement des Anliquites grecques et romaines du Nusée du Jouvre.

26 En examinant une photographie représentant la mosat̆que découpée pour son transport fig. 10), et en la comparant a une reproduction du pavement dans son état actuel, on apercoil un certain nombre de differences. La plus nette concerne sans doute les oiseaux ; le panneau superieur droil ct le panneau inférieur gauche ont éte intervertis ; des modifications asse\% importantes ont fiti apportees all champ foral dans son ensemble pour rendre les quatre oiseaux ef leur cadre paralledes a la bordure exterieure du carre central. Plusieurs des animaux entourant le buste d'Orphée ont subi des retouches figr. 11; ; si leur aspect general n'a pas changé, de nombreuses tessères modernes ont ite ajouters, en particulier pour l'animal qui se trouve on has à gauche ligre? et pour celui qui se trouve lout à fait à droite antilope. Des restaurations considerables ont ele faites sur le buste placé en haut à gauche (Saison); mais il est clair quides ont ete effectués avant linstallation de la mosaïque a Malibu : la photographie de la vente Ifearst. montre qu'elles existaient déja en 1941. On comparera celle photographie à celle qui est conservé dans les archives du Husce des antiquites nationales de saint-Germain-en-Laye, prise probahlement quand la mosaïque était encore in situ (fig. 10).

29 La mosaique fut acheté a lantiquaire A. Barsanti, a Rome, qui précise seulement qu'elle venait de la collection W. R. Hearst; la galerie Hammer de New York l'avait vendue en 1941. "Une mosaïque de pavement gallo-romaine en 22 pieces $v^{\text {e }}$ s. après J.-C. Lot 563 ".

30 Cf. G. Lafaye, Inventaire des mosaïques de la liaule et de l'Afrique, Paris, 1909, no 217.

31 Cf. B. Nogana, Mosaici antichi... del Valicamo, Rome, 1910, pl. NVIII, et M. E. Brake, Roman Mosaics of the I Ind century in Italy, dans Memoirs of the American Academy in Rome, XIII, 1936, pl. 26, 2.

32 "Sainte-Colombe, à peu de distance de la mairie, che\%. II. Chaise; 10 mai $1894 ; 12 \times 6,60 \mathrm{~m}$. Carré formé par une torsade double avec deux filets, noir et blanc. cercle en torsade noire et rouge. Six hexagones autour d'un septieme qui représente Vénus nur sur une conque, ayant a se's citis deux dauphins et trois Éros. Tigre dompté par un enfant ; trois têtes de femme (les trois Gràces ?) ; une chève noire ; un centaure. Dans les angles quatre quadriges ; sous le vainqueur, l'inscription CLXXVI." 
pavement du Musée (ietly, sans lui itre identique. Si Biebel avait pu consulter l'Ineenlaire, il aurait proposé une identification claire el assurée du lieu d'origine de la mosaïque ${ }^{33}$. La nolice $219^{34}$ du mème Incentaire en eflet résoud le problème. sous ce numéro. correspondant à un pavement trouvé aussi a sainte-Colombe mais en 1899, nous trouvons une description précise de notre mosaïque. C'est done indiscutablement à sainte-Colombe en 1899. que fut découvert l'orphée du Musée (ietty. Mais son histoire depuis celte date jusqu'a la vente Hearst en 1941 est inconnue ${ }^{35}$ el maloré l'intérèt qu'il y aurait à combler ces lacunes, c'est son étal originel qui reliendra surtout notre altention.

Placé au centre d'un pavement richement

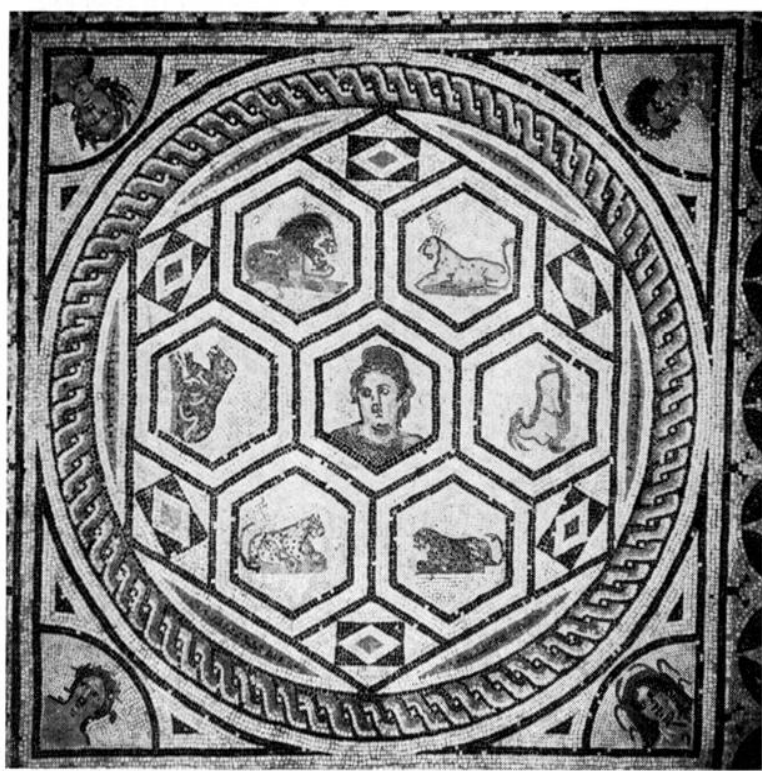

11 Délail de la même mosä̈que, alu .Huséo Paul Getly. orné apparait dans un petil hexagone le busle d'Orphée (fig. 10) : il est représenté de face. le regard tourne vers la droite. portant le bonnel phrygien. Cet hexagone est entouré de six autres. semblables: chacun deux renferme un animal dont l'espèce précise est diflicile à identifier en raison du caractire abstrait et sommaire du style de loute l'arure.

Ilais nous pourons toutefois reconnaitre nettement un lion, dans l'hexagone supérieur qauche. of en face de lui ce qui semble itre une lionne. Les animaux du bas sont probablement un ligre el une tigresse, ou des sorles de gros chats. Flanquant la figure centrale. a droite. sans doute une cheve on une anlilope. les patles antérieures croisées. I.'identification du dernier animal, à gauche d'(orphée es plus difficile: laspeet du corpse ferait songer ì un gros chien, mais c'est plus vaisemblablemenl un ours ou un sanglier que l'on a voulu representer. rar ces deux animatux accompagnent souvent Orphée.

In autre hexagone renferme le tout. inserit dans un cercle fail d'une tresse a trois brins. I'ne série de losanges ont été placés dans les angles de cet hexagone. Cette composition complexe est placce dans un cadre carré. Entre la tresse el le cadre. dans chacun des quatre angles, apparail un busle vu de face. une personnification de l'une des saisons. l'ne large bordure reclangulaire composée a partir d'un molif de greeque aver des barres verticales combiné avec le motif fréquent de la tresse encadre un champ (ouvert de rosaces dans lesquelles sont, insérées de petites croix. Dans le champ' floral, dans chacun des quatre angles, un panneau rectangulaire conlenant une figure d'oiseau (.I. STOTHART).

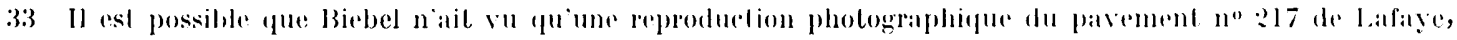

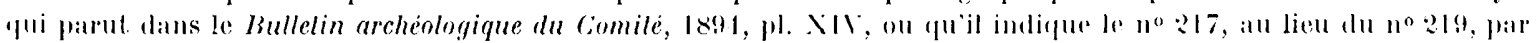
crreur salls se referer vraiment an lexte de Lafaye pour avoir une deseription complede de la mosaíque.

31 "Sainte-Colombe. Dans la propriéte de .l. Grange, sur les bords du Rhone; l 899 . Au centre, dans un compartiment hexagonal, Orphere. Toul antour, dans six autres compartiments de meme forme, lion, panthere, chivre,

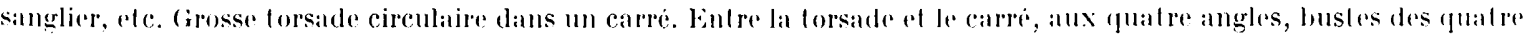
Saisolls."

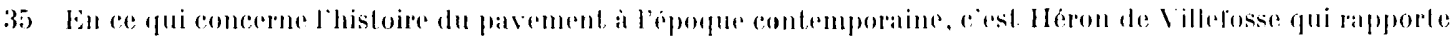

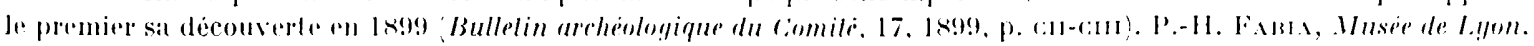

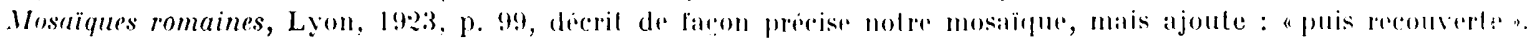

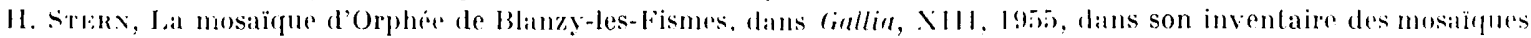

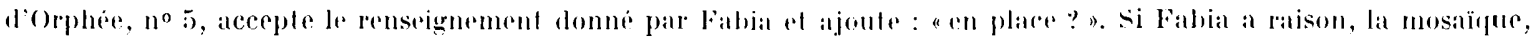

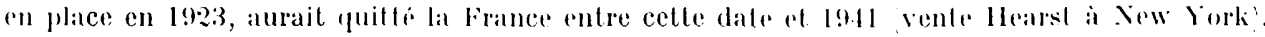


M. F. Baralte a bien voulu joindre au texte de W. Stothart la description des couleurs de la mosiäque dapres la photographie publiée dans J. P. GerTy. The .Jogs of Collecting. New York, 196i). - Orphée visage couleur chair, cheveux bruns, bonnel el lunique rouges. - Lion, pelage brun. -.- Sanglier (?). pelagre brun. - "Léopard" (en bas a gauche), pelage beige, lacheté de noir. - "Léopard" (en bas a droite), pelage brun. Lacheté de noir : contours rouges. -.- lntilope. pelage rème, contours rouges. - Faume (en haut i droite) pelage beige quelques taches grises. - Molifs geométriques complémentaires dans le médaillon. noirs et blancs, mais le losange au milieu de chaque motif (en commençant en haut et en lournant dans le sens d'une aiguille de montre) gris-verl, rouge, beige, gris-verl, rouge, beige. Tresse à lrois brins en dégrade: 1 er brin, blane, beige, rouge: 2e brin, blanc, beige, brun clair ; :3e brin, blanc, gris clair. gris fonce. - Saisons. chevelure brune, visage couleur chair: dans les cheveux. feuillages verts, plus un voile rouge pour l'Hiver. Dessin du fond, noir et blanc.

Les renseignements que ja ai pu recueillir sur la mosäque bachique du .lusée hritannique permettent de combler la lacune dans l'histoire de la mosaïque Grange qui s'ourre entre 1899 et 1941. Il est permis de penser que le pavement, en possession de Vassy et Guy en 1912 . fut proposé à la vente en Angleterre comme la mosaïque bachique. La chose est d'autant plus probahle qu une photographie aux archives du Vusée de Vienne (cliché, no 1454) (fig. 10) qui n'a pu ètre prise que lors de l'enlèvement en 1912, la montre en l'état exact, oì elle a été en arrivant au Musée Getty. M. Stothart indique en effet (cf., supra, p. 131) qu'elle a été composéc a partir de vingt-deux morceaux; or, sur la photographie

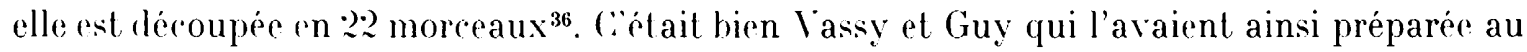
transport. Je pense qu'elle est venue de Vienne par l'Angleterre dans la collection William Randolph Hearst, d'où elle a passé dans celle de J. Paul Getty à Malibu. Cé collectionneur,

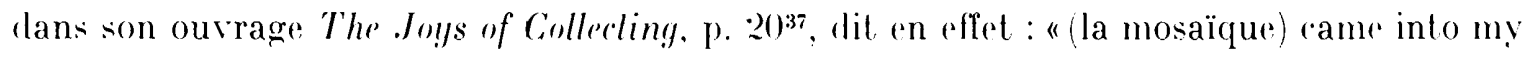
possession via the William Randolph Hearst auction held at the Hammer Gallery in New lork in 19.11 \%. Ia mention de l'antiquaire A. Barsanti de Rome38 "Purchased by A. Barsanti, W. R. Hearst Collection sale. Ilammer Gallery New York, 1941 "sexplique aisément. Ia mosaïque n'a pas été achetie par A. Barsanti pour son propre compte et acheminée à Rome, mais pour le collectionneur californien.

A l'origine cette mosaïque mesurait $6,50 \mathrm{~m} \times 4.80 \mathrm{~m}$ approximativement ${ }^{39}$, réduite maintenant a 4.95 $\mathrm{m} \times 3.43 \mathrm{~m}^{40}$. Cette réduction remonte a 1912, la photographie citée en fait foi (fig. 10). Aucune vue d'ensemble nayant ete prise sur te lieu de la fouille ${ }^{41}$ il est délicat de reconstituer les parties enlevées. Cépendant la mosaïque du Yusée britannique ayant écalement été rérluite $(2.74 \mathrm{~m} \times 2.74 \mathrm{~m}$. ('ontre $3.60 \mathrm{~m} \times 3,20 \mathrm{~m})$ par l'enlèvement des handes de raceord blanches ${ }^{42}$ il est probahle que lassy et Guy aient procédé de la même

36 An Musere J. Paul Getty, on a mis le meander de la bordure transversale superienere en bas du panneau.

37 Jr dois celte réfirence, ainsi fue colles qui suivent, a l’amabilite de .1 . F. Baralte, assistant au .Iusée du Lourer.

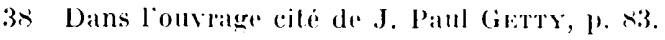

39 C.f. VAssy et fitis, o. l., p. 629 .

40 .J. Paul (ivatr, o.l., 1. 82 : " $1913 / 4$ ins. 135 ins."

11 Deux clichés aux archives du Musere de Virume les mèmes que dans l'allum, no jo, du Musere des antiquites nalionales prises probablement sur place of arant le decoupage, ne domnent que des vaes partielles.

1: Voir supra, p. 1:2. 

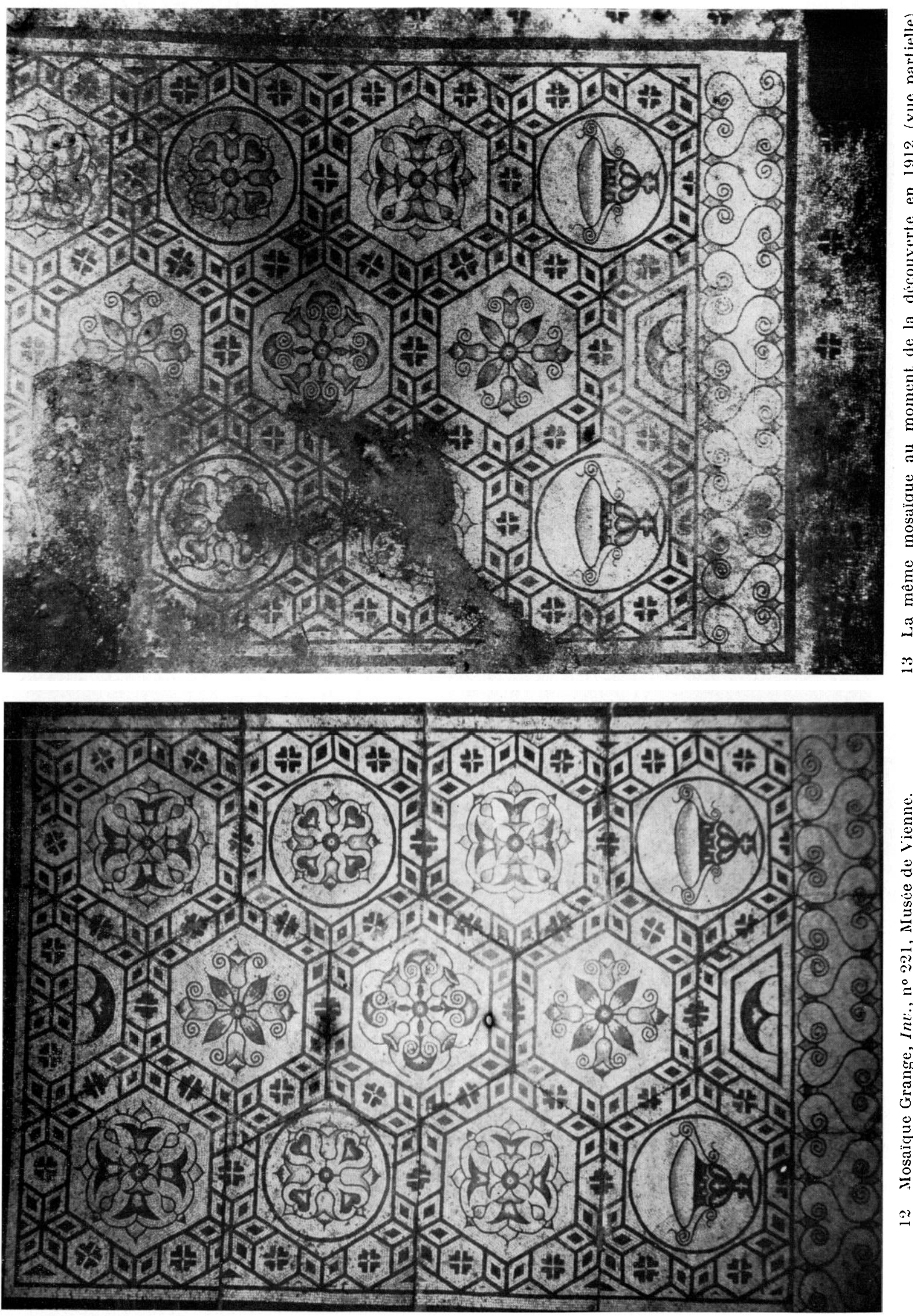


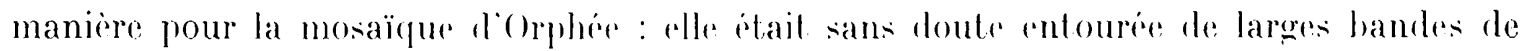
raceorl hlanches: 43 .

Franklin Biebelt4 a bien observé que le canevas de re pavement s'apparenle de près à colui d'une autre mosaïque de sainte-colombe (?). no 217 de l' Imenlaire. trouvee en 1894 l)ans les deux cas. un carré se découpe au milieu d'un fond à décor géométrique. Dans ces carré est inscrit un disque couvert de sept hexagones en nids d'abeille qui renferment des sujets figures. L agencement des hexagones vers la bordure du mélaillon est cepenclant un peu différent dans la mosäque d'Orphée. L'espace vide entre les hexagones se forme en losanges de sorte que la figure entiere devient un grand hexagone tangent a la periphépie du médaillon. I) sesisques garnis de sept nids d'abeille se trourent dans d'autres parements de la Ciaules5 qui datent du re ou du début du me siècle; un bel exemple vient d'etre public dans cettre reve 46 qui. par les délails ornementaux se plare dans l'école rhodanienne de l'extrème fin du me ou de la première moitie du me siècle. Sous reviendrons plus loin alu thème de cette mosaïque, Orphè (rharmant les animaux.

La troisieme moscïque trouvée dans la proprieté Grange, purement ornementale, qui a subi moins de restaurations que les deux autres, est entrée all Musce de Vienne, probablement en 1913. Découpée en quinze moreaux (fig. 12), alle y ast exposée, les morceaux etant mis bout a bout. L'identification du pavement est possible grâa à l’article de Vassy et Guy ${ }^{47}$. Aujourd hui la mosaïque forme un rectangle de $4,90 \mathrm{~m} \times: 3.20 \mathrm{~m}$. A l'origine elle aurail mesure $8.50 \mathrm{~m}$ sur $4.80 \mathrm{~m}$ criviron. A en croire ces mesures il y avait done en haut la mème frise de s adossés el sur les quatre còtes des bandes de raccord d'au moins $0.80 \mathrm{~m}$ de large dont les restes se voient sur notre cliche (fig. 13) /4 $^{48}$

Le canevas est a bases l'hexagones et de losanges. traces par des filets noirs sur fond blanc. Douze grands hexaggones sont groupes en quinconces par trois rangées verticales de quatre. trois et quatre. I)ans la rangée du milieu les hexagones des deux extremites sont tronqués de: moitié et devenus des trapezes. sur les cotés des grands hexagones s'appuient de petits hexagones llanqués de deux losanges. les losanges se groupent par six en étoiles, sauf vers les horls des longs cotés du champ oì elles sont amputées de la moitié el interrompues par des triangles olitus, amorees des grands hexagones. l)ans les losanges s'emboitent d'autres losanges pleins, dans les hexagones des quatre-feuilles, noirs sur fond blanc. Les grands hexagones inscrivent deux crateres et des motils floraux polpehromes rouge brique,

43 Ou garnies de quelque décor insignifiant en semis. Ins bandes de raceord de cre genre avaient eté entevées a la 3" mosaingue Grange, voir ci-dlessolis.

Hoir la notice de .I. Stothart.

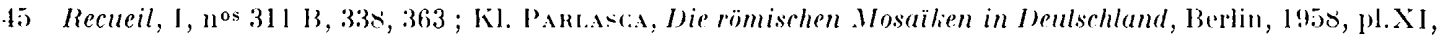

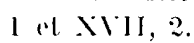

46 Supra, p. 53, fig. 4x-56.

$47 \quad 0 . l ., \mathrm{p}, 6.30$ (.t suiv.

is l.a photographie que nous reproduisons tig. 1:3 est rognee en haut. Le cliche original Vassy et Ger, fig., p. 629) comporte, au-dessus des grands hexagones de la rangée verticale du milien, l'amorce d'un quatrieme grand hexagone contenant les restes dime pelle comme celles du ge hexagone. Mne Lancha a attire mon attention sur ce detail. Il faul an conclure que la rangere centrale comportait au moins quatre grands hexigones entiers ed aux deux extremites deux demi-hexagones, celles des cotes cing grands hexagones. Toute lat partie vers le bord superieur est refinite. 
jaune ocre, gris bleu, noir) de quatre types différents : $1^{0}$ quatre calices doubles rayonnant d'un cœur circulaire sont reliés entre cux par des filaments an aceolade qui envoient une feuille lancéolée vers le centre (quatre fois). Do Quatre calices en croix reliés par des filaments alternent avec des feuilles cordiformes qui pointent vers te cour (deux fois). 30 Quatre calices rayonnant du cœur alternent avec quatre feuilles lancéolées (deux fois ; rangée du milieu). $4^{0}$ Quatre calices rayonnent du cour, des peltes sur de petits balustres s'intercalent entre eux. La rallonge est couverte d'une frise de volutes en siaceolées. Les bandes de raccord étaient parsemées à distance régulière de quatre-feuilles comme reux des petits hexagones (fig. 13).

Ce canevas revient presque identique, mais sur une surface plus large (4, 5), 4, 5) hexagones garnis de 4 cratères et de 14 motifs floraux). dans une mosaïque de Sainte-( Colombe, apparemment perdue, dont un dessin ancien (inédit) se trouve au Musée de Vienne (fig. 14); elle avait été découverte dans la "propriété Diederichs». Lees deux pavements sont sans aucun doute sortis du mème atelier. ... Le réseau d'hexagones et d'étoiles à six losanges a joui d'une certaine faveur à Vienne et, peut-être à I,yon. Ieux autres pavements. l'un trouvé à Saint-Romain-en-Gal, en 1967, l'autre découpé en morceaux et entreposé au Musée lapidaire de Vienne. tous deux inédits, le reproduisent mais sous une forme plus riche et probablement plus tardive (deuxième quart du nie siècle). L'exemplaire du Ilusée lapirlaire garde les losanges ajourés, mais garnit les petits hexagones de rosettes a six pétales banches sur fond noir. Les grands hexagones sont bordes d'un entrelacs a deux brins et inscrivent des vases, des lleurons, des fruits et des volatiles. Lexemplaire découvert récemment par II. Tourrene a saint-Romain-en-tial est exceptionnellement, somptueux : des fleurons et des sujets sinscrivent non seulement dans les grands, mais aussi dans les petits hexagones. L'échantillon de Lyon ${ }^{49}$ est proche de celui du llusée lapidaire de Vienne. I'n autre. d'Auriol (Bouches-du-Rhòne) so, s'apparente également aux exemplaires de cette ville, alors que deux pavements d'Aquitaine ${ }^{51}$ s'en distinguent par des détails.

Les motifs de remplissage du groupe, cratìres, tleurons et sujets, s'appliquent à Vienne. à la fin $\mathrm{du}_{11}{ }^{\mathrm{e}}$ et au $\mathrm{II}^{\mathrm{e}}$ siècle, aux ('anevas les plus divers ${ }^{52}$. Les volutes en $\mathrm{S}$ arlossées. relativement rares, forment la rallonge d'une mosaïque de Lyon ${ }^{53}$ et le cadre d'une autre de cette ville ${ }^{54}$.

Résumons nos observations: les trois mosaïques (irange sont d'un caractere typiquement rhodanien, de la fin du ${ }_{1}{ }^{\mathrm{e}}$ ou du tout début du ${ }_{11}{ }^{\mathrm{e}}$ siècle. Elles s'ajoutent à de. nombreuses autres pour compléter le tableau qui commence a se dessiner du style, tries marqué, des ateliers de Vienne.

19 Recueil, II, no 99.

50 Inveniaire, no 64.

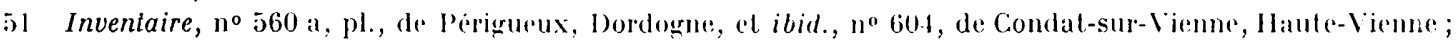

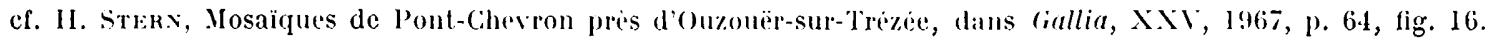

52 Cf. par ex. la mosaïque de Lirresse d'IIercule, H. Strks, dans Monumenls l'iol, l.c., el d'autres.

53 Recueil, II, no 42 .

54 Ibid., no 136 ; sur l'histoire du motif, ibid., p. 11. 


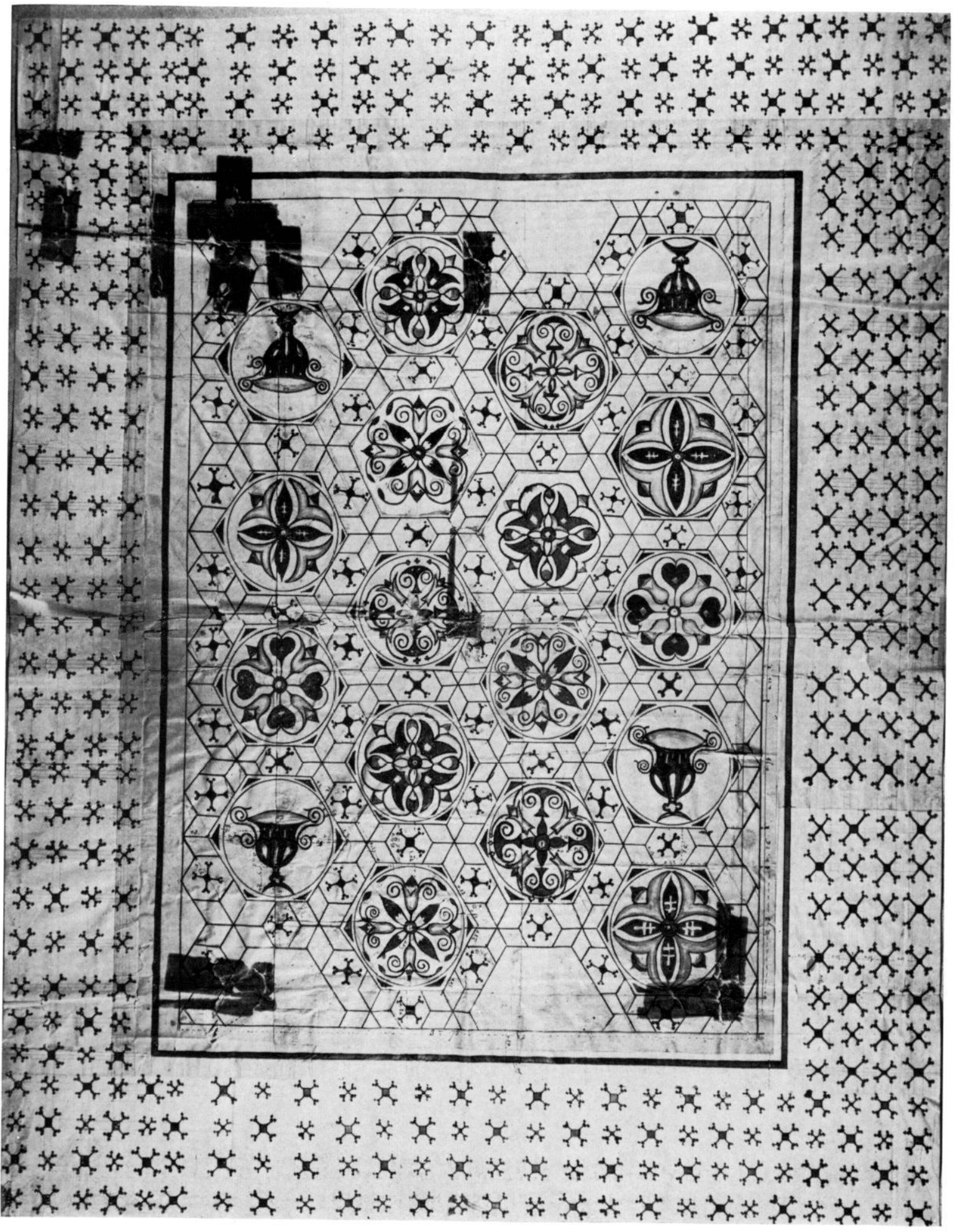




\section{La mosaĭgue Montant.}

Le destin a été moins favorable à une seconde mosaïque d'Orphée, découverte en octobre 1822 à Saint-Romain-en-Gal, dans la vigne d'un certain Iontant, dit Paret ${ }^{55}$. Achetée, en mai 1823, par la ville de Lyon ${ }^{56}$, elle ful exposée dans une salle du Musée des Beaux Arts de cette ville où elle resta jusqu'en 1954. Entoilée elle fut alors transportée chez le mosaïste Jouvray de Lyon, où elle séjourna jusqu'en 1968, année où elle fut détruite dans un incendie qui ne laissa subsister que trois morceaux.

Ph. Fabia a relaté avec soin la découverte de cette mosaïque, les vicissitudes de son histoire (jusqu'en 1923) et la réduction qu'elle avait subie pour ètre exposée au Musée de Lyon. Pour en restituer l'état originel il s'appuyait sur quelques notices plus ou moins contemporaines de la découverte, sur une aquarelle de F. Artaud ${ }^{57}$ (fig. 15), sur un dessin du même auteur ${ }^{58}$ et sur la mosaïque elle-même, conservée alors au Musée (fig. 16). Un lot d'aquarelles dans un recueil factice aux archives du Musée de Vienne représentant 29 animaux, sans doute la totalité de ceux qui étaient conservés, lui avait échappé (fig. 17 à 28). Nous nous en servirons pour donner une reconstitution de l'état du pavement au moment de la découverte.

Peu de temps après la trouvaille, en décembre 1822 ou en janvier 1823, Artaud se rendit sur les lieux et fit dessiner la mosaïque ${ }^{59}$. Les aquarelles en question sont certainement le produit de ce travail, exécuté sur place. Elles sont portées sur les feuillets d'un cahier de dessin de $162 \times 228 \mathrm{~mm}$ dont un seul, marqué du $\mathrm{n}^{0} 9$ (invisible sur la fig. 25) et donnant cinq animaux, est intact. Les autres ont été coupés en trois morceaux, numérotés de 1 à 8 et de 10 à 12 (une partie seulement de ces nos est visible sur nos clichés); chaque feuille découpée reproduit deux ou trois animaux, une autre l'image d'Orphée. Ces images portent des numéros d'ordre qui permettent de restituer la séquence primitive.

Les dimensions de la mosaïque au moment de la découverte étaient évaluées à $20 \times 15$ pieds, y comprise la bordure, un mince rinceau d'acanthe (fig. 15). Le champ était divisé en $8 \times 6=48$ octogones, dont les quatre du milieu étaient tronqués par le tableau carré d'Orphée. A l'origine quarante-quatre animaux différents étaient inscrits dans les octogones ${ }^{60}$. Un certain nombre d'octogones étant perdus, d'autres en mauvais état, il était impossible d'exposer la mosaïque entière. Selon la mode du temps on préférait éliminer un grand nombre de caissons conservés pour présenter un pavement intact. On le réduit à douze caissons et au tableau d'Orphée, à l'aide de morceaux originaux (fig. 16). Deux octogones, contenant un lion et une chouette furent utilisés, l'un en 1868 pour décorer le mur du "Vestibule des Antiques" (depuis 19\%4 secretariat) du Musée des Beaux Arts,

55 Voir Ph. Fabia, Mosaïques romaines des . Musées de Lyon, Lyon, 1923, p. 83 et suiv.

G. IAFAYE, dans l'Inventaire, a dédoublé cetle mosaïque en la faisant figurer aux nos 201 et 2.42 .

56 Ph. Fавік, o.l., p. 88. I a dépense a été de 6625 frs.

57 Histoire abrégé de la peinture en mosä̈que..., I,yon, 18:35, pl. I.VIII, texte, p. 119 et suiv.

58 Ph. Fabia, o.l., p. 87, fig. 9.

59 Ibid., p. 85.

60 Fanis, o.l., p. so et suiv, a montré que la mention, par divers auleurs contemporains, de 50 caissons est erronée. 


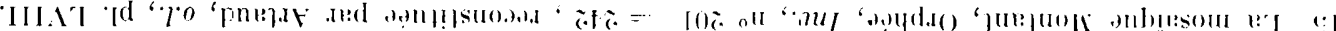

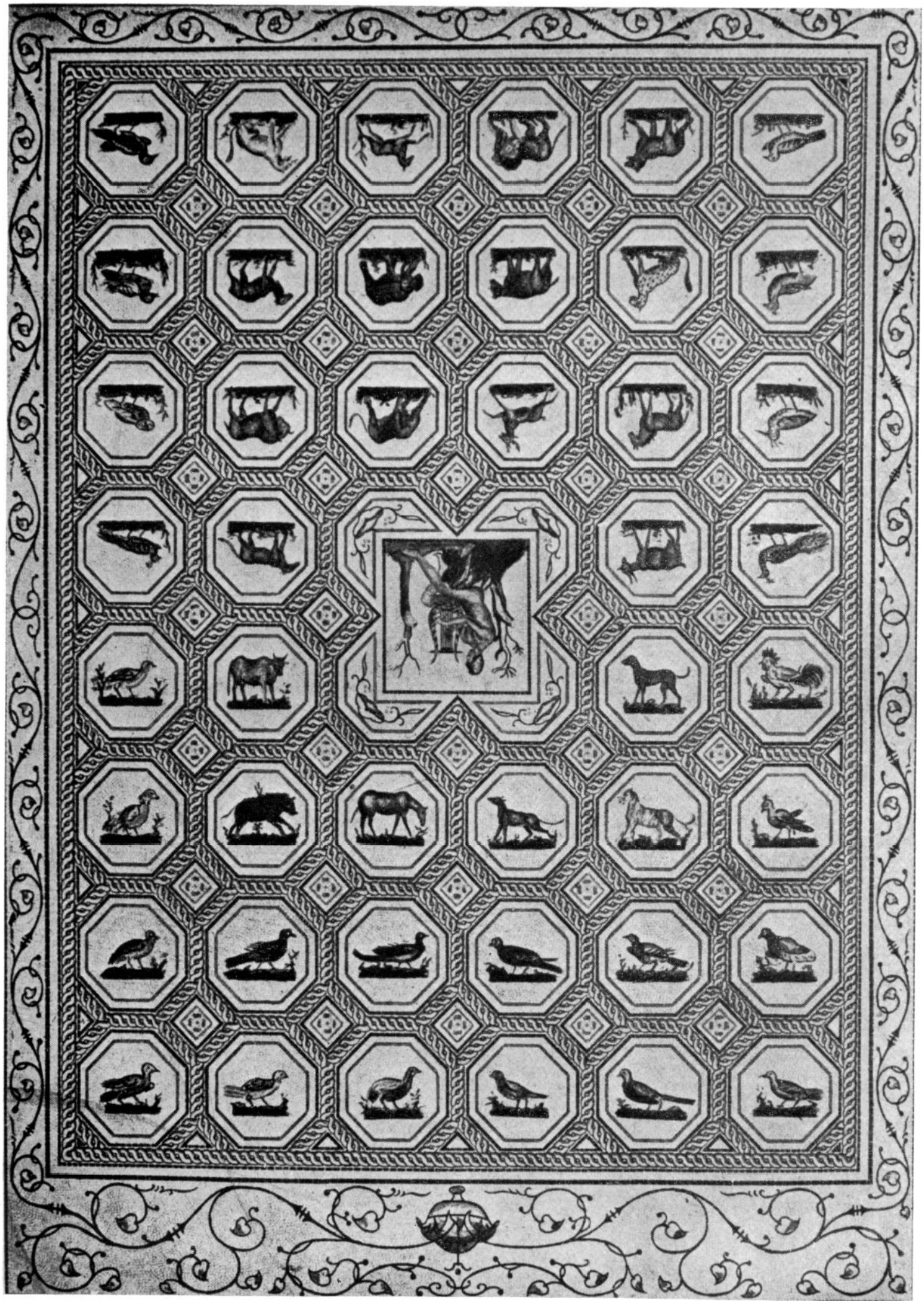




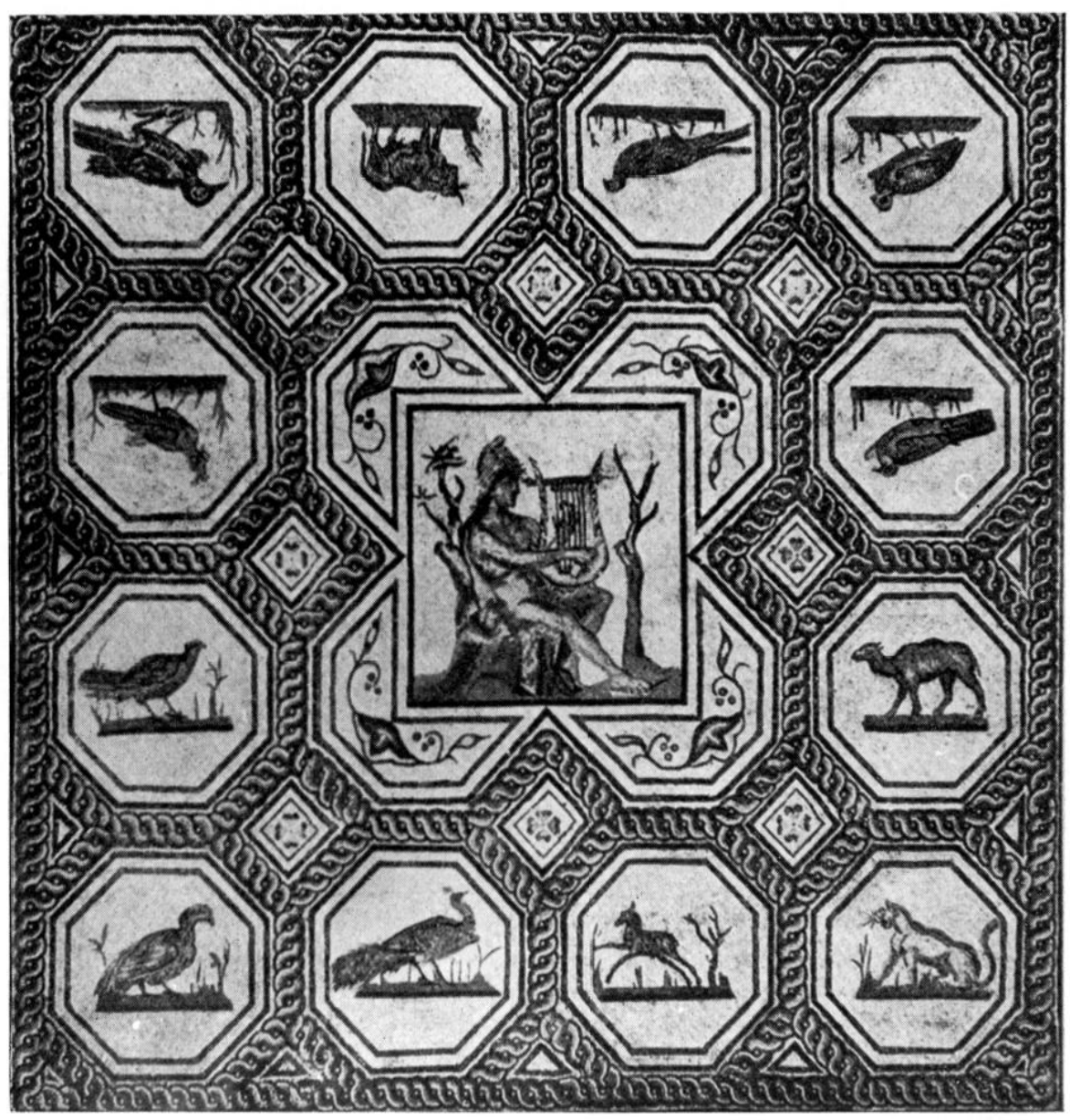

16 La même mosaique, au .Musée des Beaux-Arts de Lyon. D'après la planche de l'Inventaire.

l'autre en 1877 pour orner le mur d'un couloir au rez-le-chaussée du Musée ${ }^{61}$. transformé en 1935 en petite pièce d'exposition de sculptures romaines. Cee dernier a été jeté aux déblais en 1935, le lion est conservé (fig. 34), mais actuellement invisible dans les réserves du futur Musée des antiquités gallo-romaines de Lyon. Les quinze caissons restants semblent avoir disparu peu de temps après la découverte.

Les animaux de nos aquarelles sont numérotés de 1 a 3,5 à 11.13 a 19, 21 a a 31 et:37; leur séquence, sans être rigoureuse, suit un certain ordre logique. Eille commence en bas à l'angle inférieur de droite (fig. 29a) pour remonter clans la rangée verticale de ce côté; le no 4, perdu, étail compté. I)ans l'angle supérieur elle tourne vers la gauche comprenant le no 9. Mais au lieu de continuer dans la rangée transiersale supérieure elle descend verticalement à l'avant-dernière rangée du haut $\left(n^{0} 10\right)$ et se poursuit dans cette rangée jusqu'au $\mathrm{n}^{0} 14$ en comptant le no 12 qui était perdu. 1), là elle tourne à angle droit dans la rangée extérieure de gauche en omettant le caisson entre 14 et 15. Arrivée à l'angle inférieur gauche

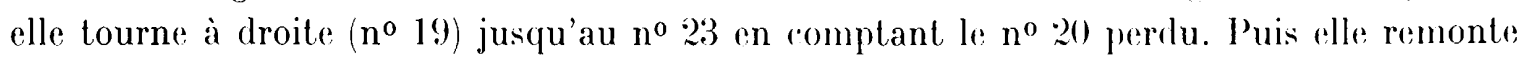




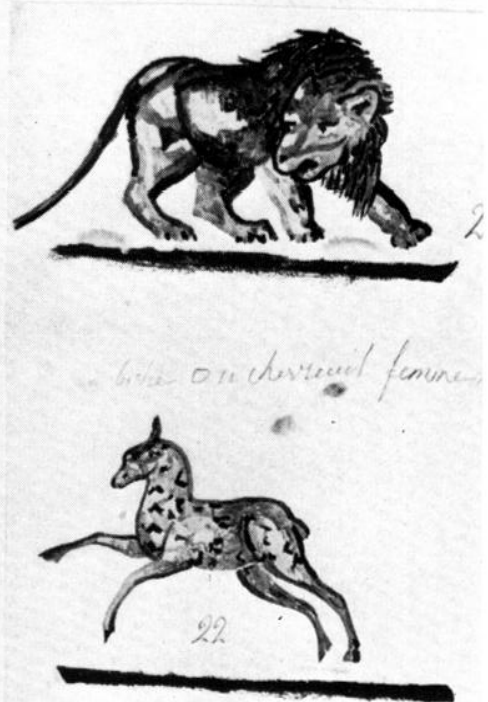

17 Lion, biche.

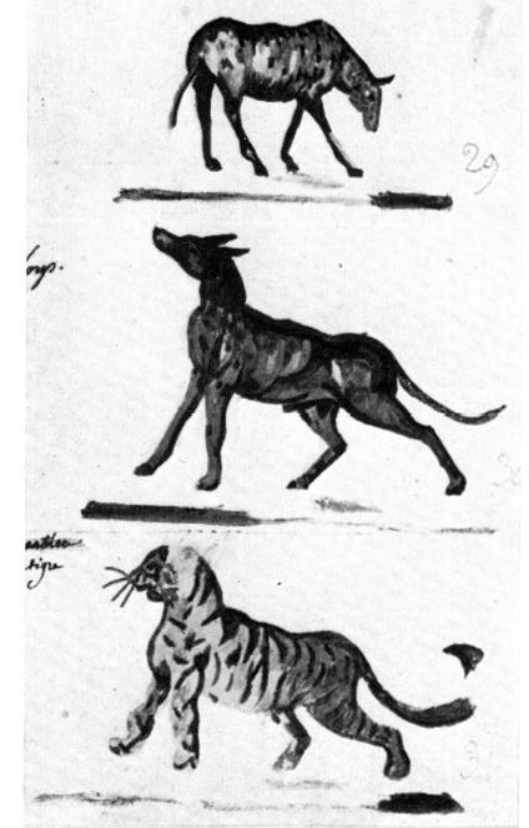

21 Agneau, loup, tigre.

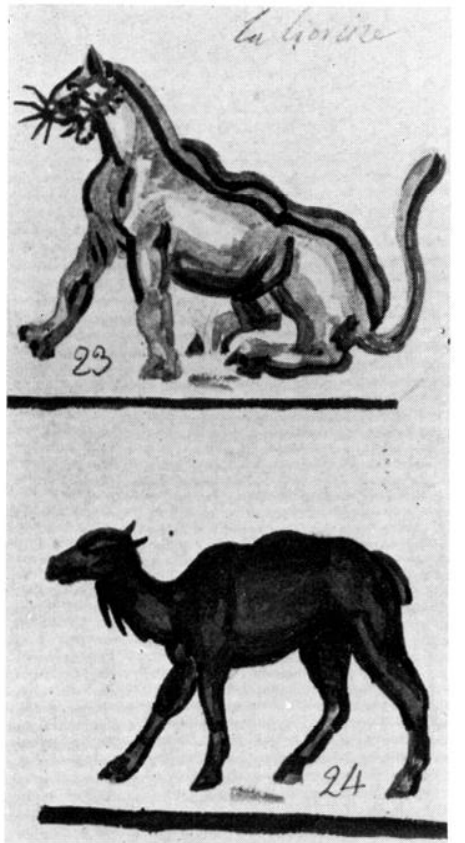

18 Lionne, chameau.

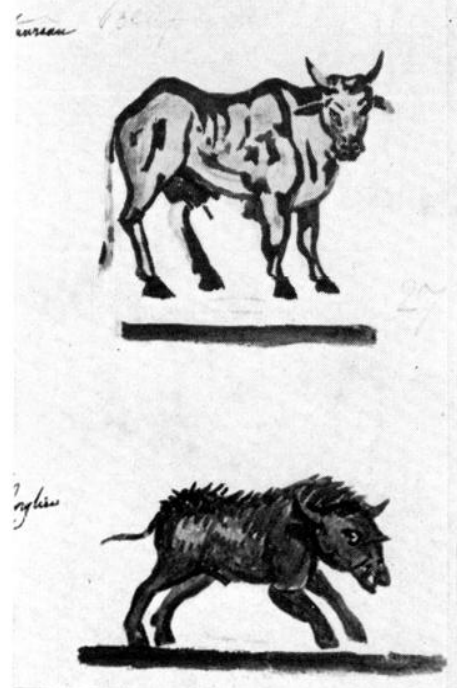

$20 \quad$ Vache, sanglier.

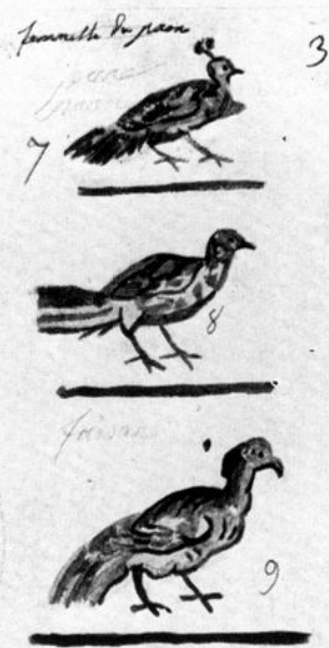

19 Paonne, oiseau, faisan.

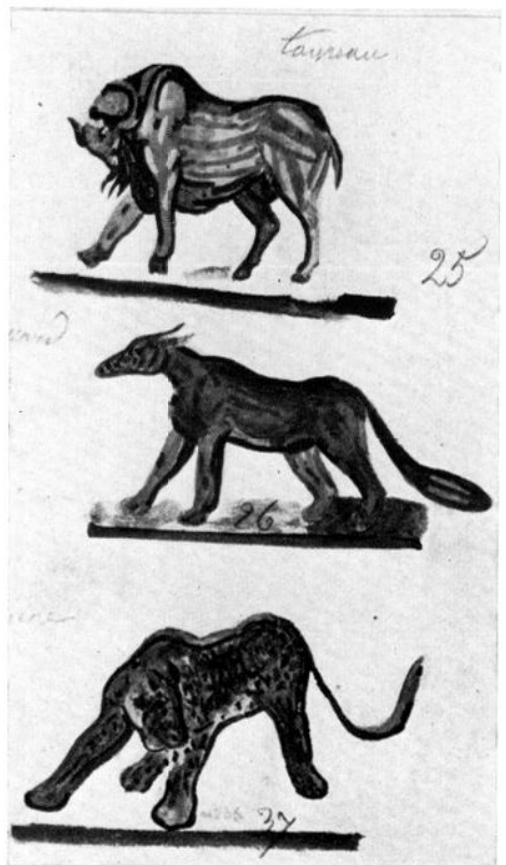

2: Taureau, renard, panthère.

17 à 28 Aquarelles des animaux de la mème mosaïque, exécutes en 1×23, Murée de Vienne.

pour tourner autour du tableau central dans le sens contraire à l'aiguille d'une montre jusqu au no 31, compte les caissons 32) à 36 qui étaient perdus et atteint le no 37 (panthère) que jaai placé clans la deuxième rangée transversale du bas. mais qui. peut-être, se trouvait au-dessus ${ }^{62}$. Cés emplacements correspondent à ceux de la planche IXIII d'Artaud (fig. 16)

6? c.f. la pl. d'Artaud ; fig. 15 ). 


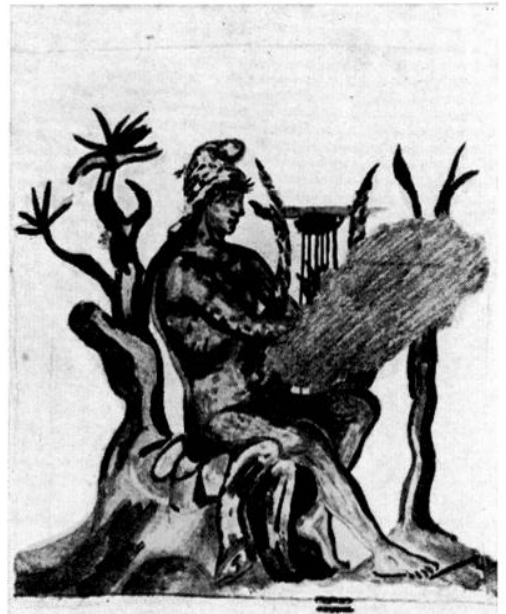

23 Orphée.
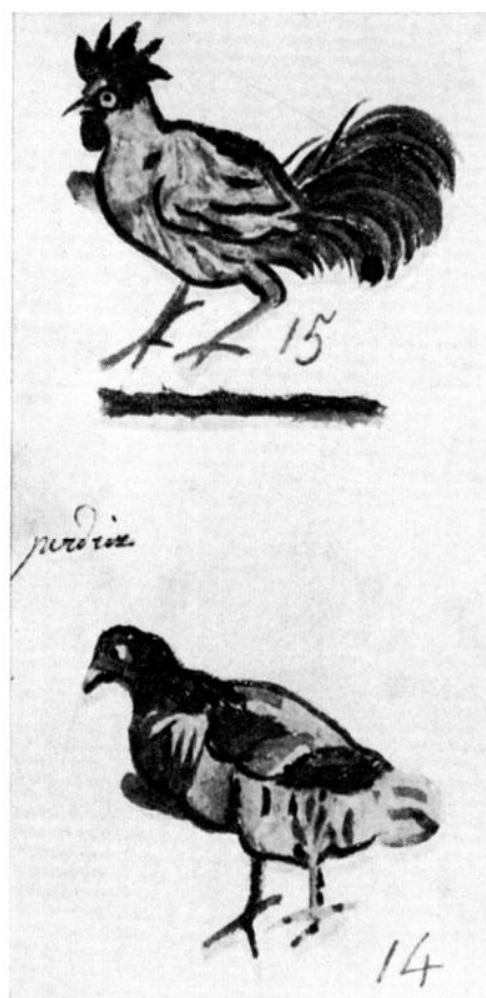

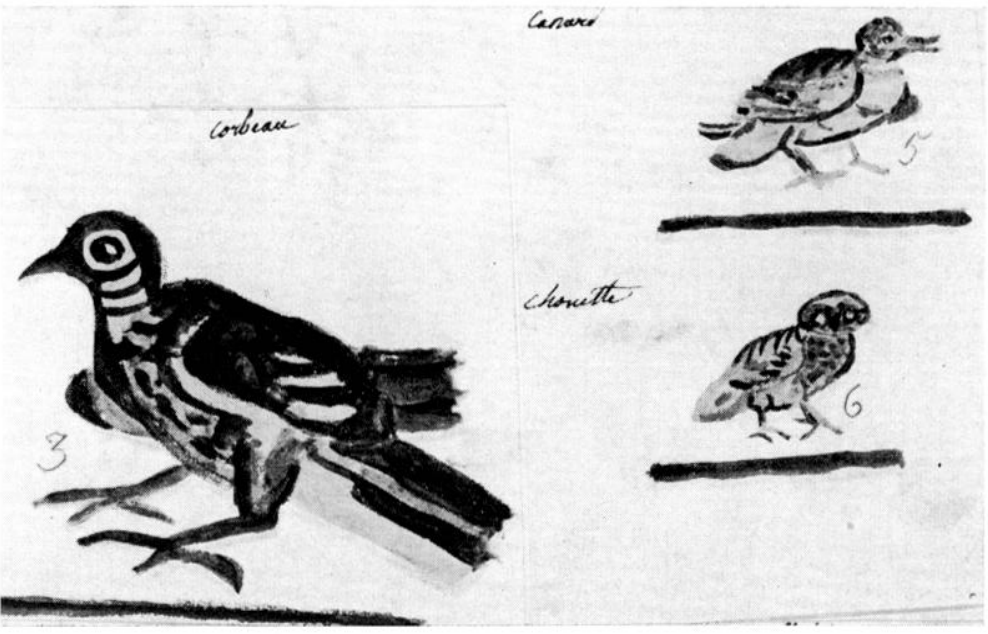

24 Canard, chouette, corbeau.
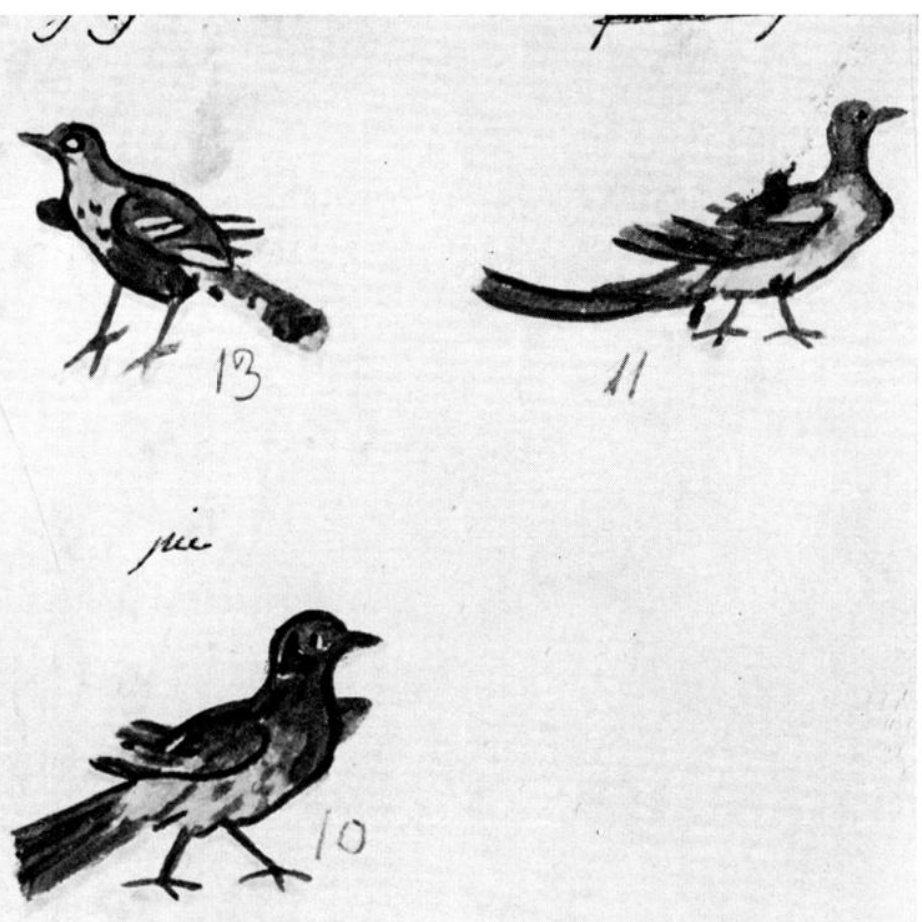

25. Coc, geai, oiseau indetermine, perdrix, pie.

sauf ceux des nos 1 (perdrix?) (fig. 27). 2) (perroquet) (fig. 28). 3 (corbeau), 5) (canard), 6 (chouette) (fig. 24), et 7 (paonne) (fig. 19), 5) et 6, orientés vers l'extéricur ont été descendus dans 2 et 3 , et renversés pour regarder vers l'intérieur, 7 a été placé dans 4 et également renversé $e^{63}$. Le corbeau, descendu du $n^{0} 1$, le $n^{0} 1$ remonté au $n^{0}$ après 9 et le perroquet au $\mathrm{n}^{0} 12$, ont gardé leur orientation primitive.

63 liexactitude de l'orientation de la chouette dans notre aquarelle est confirmée par les indications de fabia (o.l., p. 88) qui a vu l'original. 


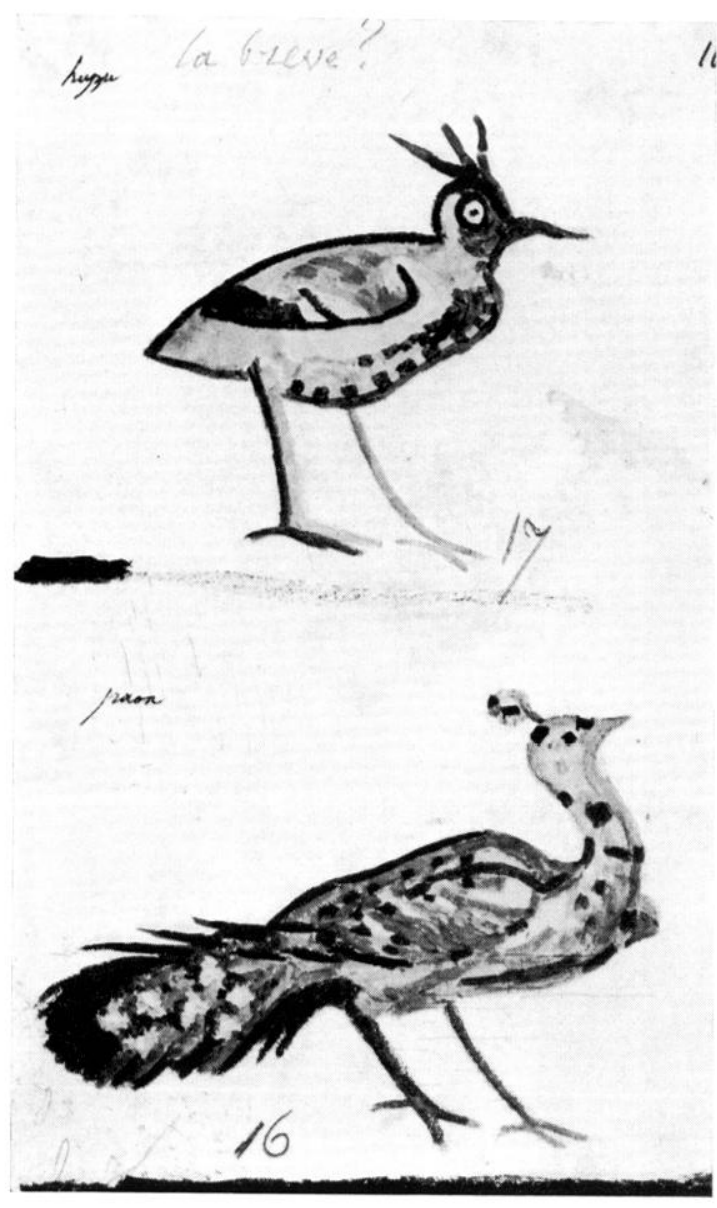

26 Huppe, paon. l6 $\quad$ /1
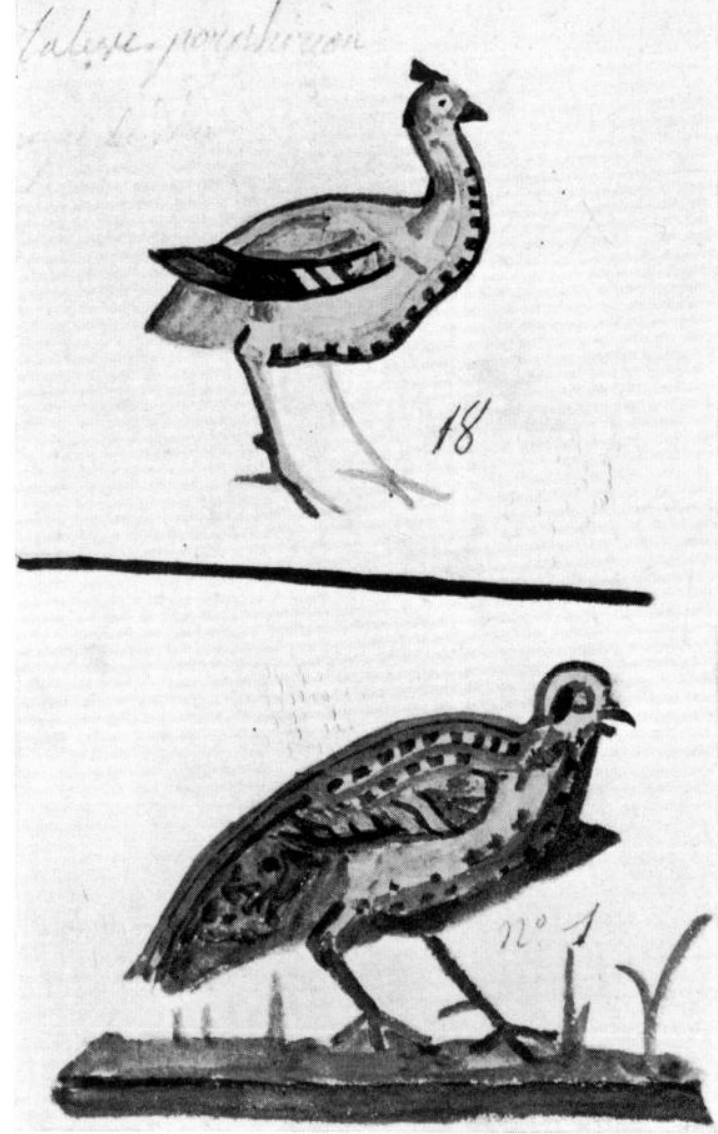

27 Dinde, perdrix.
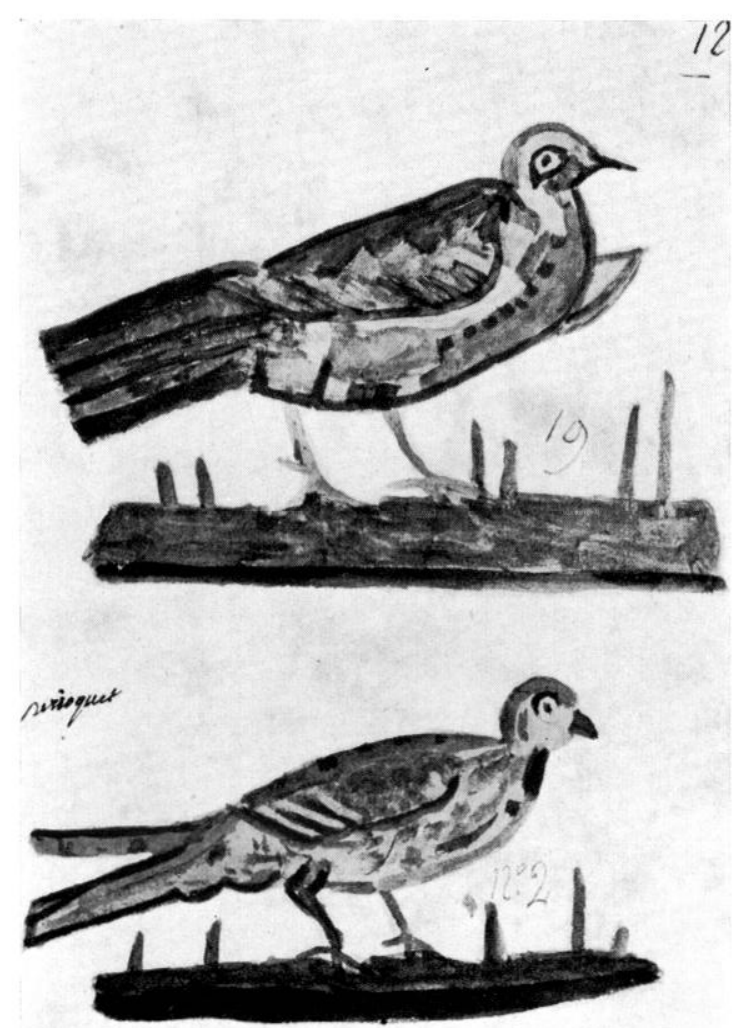

28 Oiseau indéterminé, perroquet. 


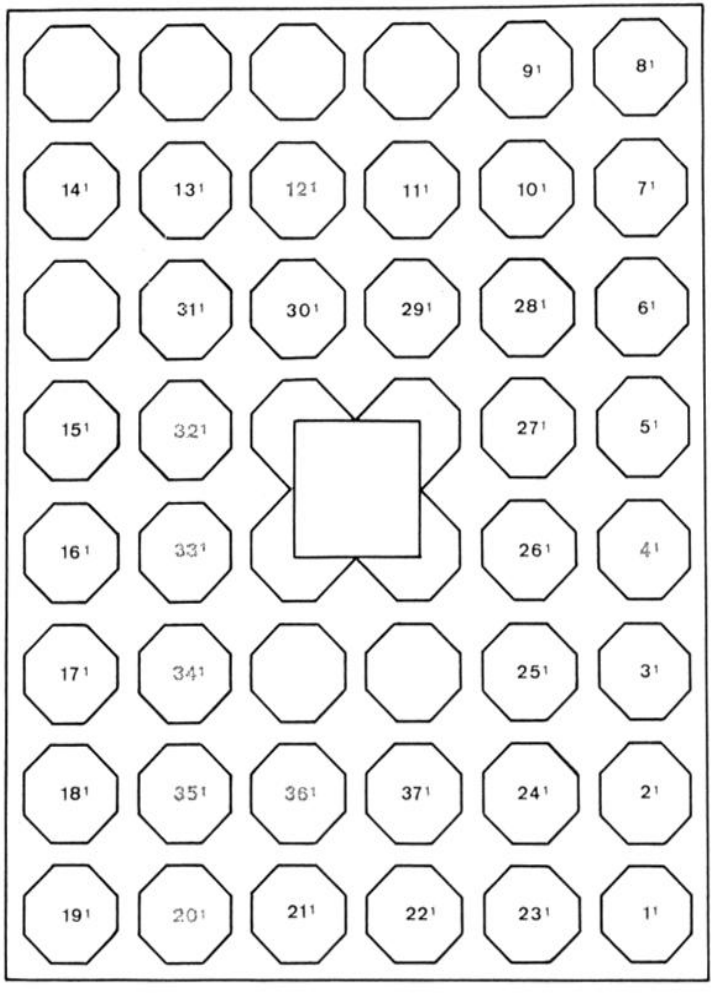

1

29a Schema de la mème mosaique suivant la numerotation des aquarenles.

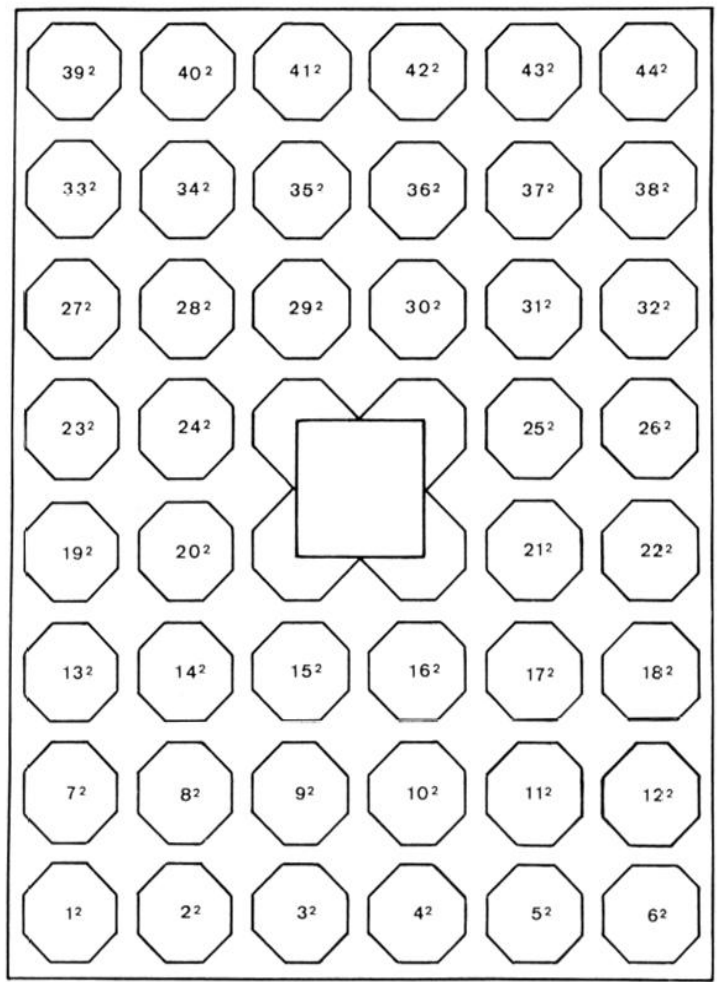

2

:(1) Schema de la mème mosäque suivanl notre numérotation.

Pour rendre plus aisée l'étude qui suit nous conterons les caissons de $1^{2}$ à $11^{2}$ commentçant en bas à gauche et finissant en haut a droite, en passant dans chaque rangée transversale de gauche à droite $\left(1^{2}\right.$ à $6^{2}, 7^{2}$ a $12^{2}$, etc.) (fig. 294 ). Notre reconstitution (fig. 30) basée sur la numérotation des aquarelles du Ilusée de Vienne montre deux grandes brèches qui s'ouvraient dans le pavement, l'une en haut à gauche (caissons nos $39^{2}$ à 1922 ), l'autre le long du tableau d'Orphée (caissons nos $2^{2}, 8^{2}, 92,14^{2}, 15^{2}, 16^{2}, 20^{2}, 24^{2}$, et $\left.27^{2}\right)$, enfin une lacune a la place du caisson $n^{0} \cdot 2^{2}$ à droite. C'est sans doute ainsi que la mosaïque avait eté trouvée.

Artaud (fig. 15) a dessine dans les vides des animaux de son invention, un rhinoceros $\left(n^{0} 2^{2}\right)$, une panthère $\left(n^{0} 8^{2}\right)$, un ours $\left(n^{0}\left(9^{2}\right)\right.$, un cheval $\left(n^{0} 14^{2}\right)$, un cerf $\left(n^{0} 15^{2}\right)$, un eléphant $\left(\mathrm{n}^{0} 16^{2}\right.$ ou $\left.10^{2}\right)$, un boue $\left.\left(\mathrm{n}^{0} \cdot 2\right)^{2}\right)$, un chien $\left(\mathrm{n}^{0} 24^{2}\right)^{64}$ at six oisealux $\left(\mathrm{n}^{\text {os }} 27^{2}, 33\right)^{2}, 39^{2}$ a $\left.42^{2}\right)$. Son procédé est facile a comprendre. Voulant montrer au lecteur un pavement intaret il a choisi des quadrupèdes qui habituellement figurent dans les tableaux antiques d'orphée charmant ies animaux et dont il etait d'ailleurs aise de trouver des modiles. Au contraire les oiseaux nos $26^{2}, 32^{2}$ à $38^{2}$ et $399^{2}$ à $41^{2}$, inspirés par des images existantes $\left(392^{2}\right.$ a $41^{2}$ par $31^{2}$, $26^{2}$ et $38^{2}$ par $6^{2}, 32^{2}$ par $\left.19^{2}\right)$ ont peu de caractère et ne prétendent pas à représenter des espèces

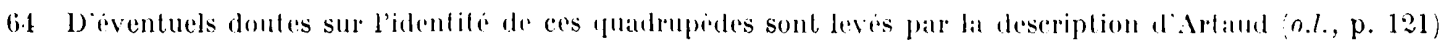
qui les énumère nommiment. 
définies. Il est plus difficile de romprendre les déplacements des autres volatiles. Celui de la perdrix (notre $n^{0} 6^{2}$ ) sexplique : passéc à $12^{2}$ elle prenait une orientation correcte. Mais il a fallu inverser le perroquet (passé de $12^{2}$ à $35^{2}$ ), la paronne qui vient de $38^{2}$ à $22^{2}$, la chouette qui descend de $32^{2}$ a $18^{2}$, le canard qui est déplacé de $26^{2}$ a $12^{2}$ (le corbeau passant de $18^{2}$ à $6^{2}$ garde son orientation). La paonne a été déplacée et retournée pour faire face au paon à l'autre extrémité de la rangée horizontale $\left(\mathrm{n}^{0} 19^{2}\right)$. Le déplacement et partant le renversement de la chouette et du canard s'expliquent peut-être par l'intention de montrer sur la partie principale de la mosaïque des volatiles particulièrement caractéristiques; celui de la perdrix, mise à côté du faisan $\left(\mathrm{n}^{0} 43^{2}\right)$ par l'idée de grouper ensemble du gibier a plumes.

Valgre ces modifications Artaurl a respecte les grandes lignes de la composition. Les volatiles occupaient les deux rangées verticales extérieures et les deux rangées transversales du haut (en comptant, bien entendu, les caissons extrèmes dans les unes et les autres), les quadrupedes. les caissons autour d'Orphée et les deux rangées transversales du bas (moins les caissons des extrimités). Tous les animaux sauf la perdrix et le perroquet étaient orientes vers les axes vertical et horizontal du champ, de maniere à tourner leur tête vers le chantrete.

Ce groupement des animaux el certains agencements de détail, l'agneau entre le loup et le sanglier66. la biche entre le lion et la lionne, prouvent que le mosaïste a bien voulu montrer les effets apaisants de la musique du chantre, malgré l'isolement des animaux ${ }^{67}$.

Parmi les neuf mosaïques d'Orphée trourées en France ${ }^{68}$ les trois exemplaires de la région de Vienne. la mosaïque Grange, la mosaïque Montant et Inv. $\mathrm{n}^{0} 181$ (=233) sont du type géométrique qui isole les animaux. L'adaptation du thème au schéma du fond est à peu prés réussie dans les deux premières. I lans la mosaïque Inv. no 181, le désordre actuel est probablement le résultat d'une recomposition toute provisoire ; on s'est contenté de rapprocher les morceaux découpés lors de l'enlèvement. - Nous avons signalé la fréquence du canevas du fond de l'Orphée Grange en Gaule. Le réseau d'octogone et de petits carrés, bordé d'entrelac's, de la mosäque. Iontant, dont celui du no 181 n'est d'ailleurs qu'une variante, a été également en faveur clans la vallée du Rhòne69.

Les personnifications des saisons placées autour du chantre sur la mosäque Grange

65 Dans le projet de reconstilution dessine par. Artaud, cf. FABIA, o.l. p. \&7, figr. 9, ce principen'est pas respecte, mais on yest rerenu, dans la réfection du Musie des Beaux-Arls, pour laquelle douze caissons originaux avaient éte utilisés. En y ajoutant la chouetle ot le lion, on obtient quatorze : quinze caissons avaient donc été écartés. Selon Fabia (o.l., p. 88) la restitution de la mosairgue entiere aurait coùti 14972 frs, plus que le double du prix du travail effectivement exécuté. C'était sans doute ce coût élevé qui aviat provoqué la réduclion considérable.

66 Déja releve par Artaud, o.l., p. 1:1, n. 1.

67 cif. H. Strers, Ia mosä̈que d'Orphée de Blanzy-les-Fismes, dans fiallia, NIII, 1955, p. 41 a 77 . Aux 17 mosaïques d'Orphé réunies dans cet article, il faut en ajouter une vingtaine venuts an jour depuis quinze ans. J'en traiterai dans une ilude en préparation sur l'iconographie d'Orphée.

$68 \mathrm{~J}$ en ai cite huit dans l'article mentionné ci-dessus. Il fant ajouter une neuvieme, trouvé à Saint-Paullès-Romans, localitie située à une soixant ane de kilomettres à l'est de Vienne, cf. Gallia, XXVI, 1968, p. 594-595, sans photographie.

69 Cf. Lne mosalique de Virmue, Ime'., no 196, deux do Nimes, Ime, nos 287 et 314 a. - Mme V. vov

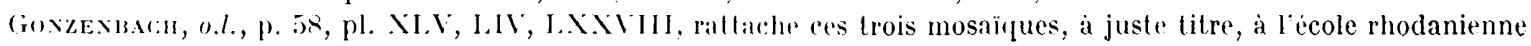
ses references aux $n^{\text {os }}$ de l'Invenlaire sont erronées'. 


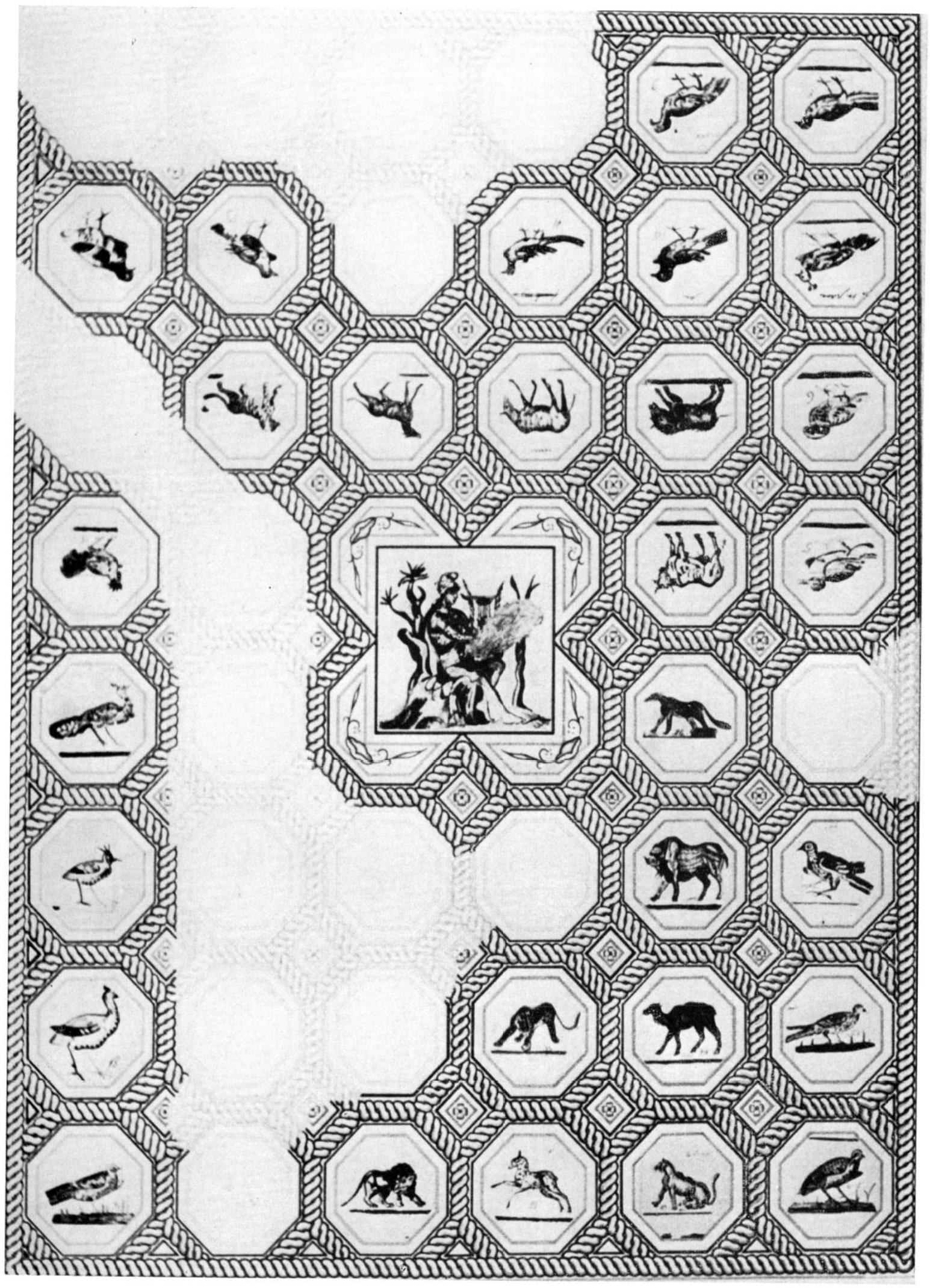

30 Ja mème mosiäque reconstituer a laide des anfuarelles. 
trouvent un parallèle dans un autre pavement gallo-romain ${ }^{70}$ et dans une mosaïque de Grande-Bretagne ${ }^{71}$. Sans avoir probablement le lien spécial avec Orphée que leur attribue M. Hanfmann ${ }^{72}$ elles précisent le ròle du chantre qui est censé apporter la paix universelle, symbolisée par l'apaisement des bètess sauvages. Les images saisonnières confèrent à son action une dimension cosmique.

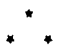

Après avoir rédigé les notes qui précèdent j’ai pu obtenir les photographies de trois octogones de la mosaïque Montant qui ont échappé au feu. Entoilés, ils se trouvent actuellement pour restauration chez le mosaïste (C. Bassier à Périgueux qui a bien voulu m'en faire parvenir les prises de vue que je publie (lig. 31 à 33). I)e l'octogone contenant le lion (notre $\left.n^{0} 3\right)$ que j’avais vu il y a sept ans au dépôt de llagneval, à Lyon, je joins une photographie que j'avais prise lors de cette visite (fig. 34).

Les trois octogones sauvés du feu, la lionne (notre $n^{0} 5^{2}=$ aquarelle Artaud $n^{0} 24^{1}$ ), le geai $\left(\mathrm{n}^{0} 34^{1}=\mathrm{A} 13^{2}\right)$, et le faisan $\left(\mathrm{n}^{0} 43^{2}=A 9^{1}\right)$ ont été récupérés en mauvais état. Tels qu'ils apparaissent sur les clichés ils donnent peu de prise à une analyse du style. D'après les renseignements fournis par M. Bassier, ils mesurent environ $0,70 \mathrm{~m} \times 0,60 \mathrm{~m}$. Parmi les cubes il y a des blanes, des noirs, des verts, des rouges, des jaunes et des roses; tous sont de calcaire, sauf les roses qui sont de marbre. Ces animaux sont rendus avec assez de bonheur. La lionne ouvre la grueule pour rugirir, alors que les volatiles se tiennent immobiles, comme figés par l'effet de la musique. Ces attitudes sont habituelles dans les mosaïques d'(Orphée.

Le lion, dont Fabia affirme qu'il a cté remis à neuf en 1868 lors de son utilisation dans le décor du "Vestibule des Antiques "73 est un bcau morceau (fig. 34). Domplé par une force invisible, le fauve est comme en arrêt, rugissant comme la lionne devant lui. Le style est pictural, le modelé rendu par des taches de couleurs et de vigoureux rehauts de lumière, l'épaisse crinière de teinte sombre est parcourue de stries brun-noir.

En me limitant parmi les innombrables représentations de lions des mosaïques romaines à celles de la Gaule et des deux Germanies, je ne trouve que deux qui, par l'attitude, sinon par l'expression de la bête, se comparent à la nòtre. Sur la mosaïque de Nennigia un lion du cirque, tenant une tète d'âne sauvage sous une patte avant, retourne la tète un peu comme relui de Vienne. Mème mouvement du lion qui sert de monture a un petit amour dans la mosaïque dionysiaque de Cologne $e^{75}$, mais l'expression du lauve est bonnasse et presque raricaturale. Un lion d'une mosaïque de Vaison-la-Romaine, publiée par J. Jassus ${ }^{76}$, vu entièrement de profil, est conventionnel, de mème que le lion bondissant de la mosaïque:

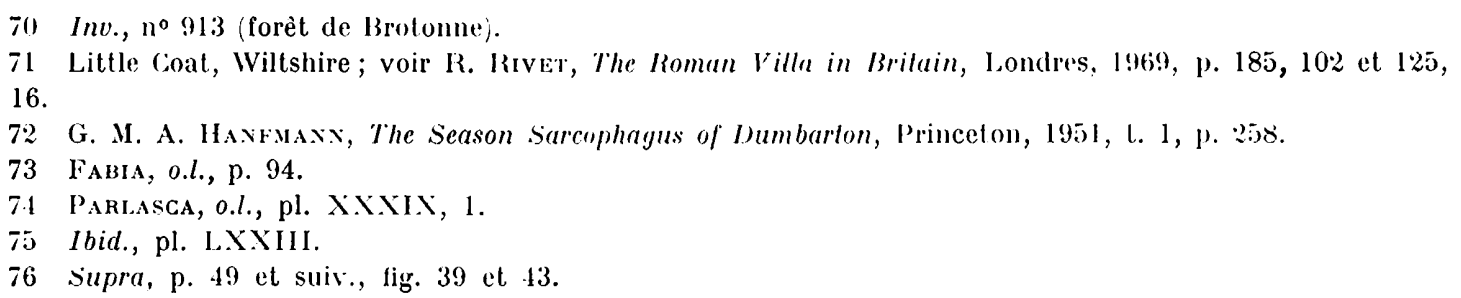


d'Orphée de la Forèt-de-Brotonne? par un dessin sommaire.

Le type du nôtre est sans auc!nn doute un cliché largement répandu dans la mosaïque romaine. L'un des plus beaux exemplaires a été trouvé de l'autre côté de la lléditerranée, en Tunisie, dans la maison de la procession dionysiaque d'El-J em7. Comme à Cologne, la bète sert de monture, mais a un Ijionysos enfant. Elle avance en sens inverse de la nôtre et baisse la tète en la retournant, la gueule ouverte. Le style est différent, moins pictural qu'à Yienne, la plasticité des formes est rendue avec soin. les transitions des ombres aux lumières sont délicates. L. Fourher date cette mosaïque du milieu du ne siècle, Kl. Parlasca celle de Nennig des années trente du ${ }_{11}{ }^{\mathrm{e}}$ siècle. celle de Cologne vers 220. La nôtre, sans que la comparaison du style avec ces deux pavements rhénans soit décisive, en est sans doute plus proche que de l'œurre a fricaine. Le schéma ornemental de la mosaïque IIontant, que l'étude systématique des mosaïques de Vienne permettra probablement de classer ('hronologiquement, me semble suggérer ces mèmes dates, c'est-à-dire le deuxième quart du III $^{\mathrm{e}}$ sicole $^{80}$.

\section{Henri Strkx.}

77 Inventaire des mosaïques de la liaule, $n^{\circ} 913, \mathrm{pl}$.

78 Recueil, I, $n^{\circ} 346 \mathrm{~A}$.

79 Cf. L. Foc ChER, La maison de la procession dionysiaque à El-.Jem, Paris, 1963, p. 119, fig. 17.

80 Ies clichés des fig. 1, 2, 3, 1, 10, 12, 13 ont eté communiqués par le Musée de Vienne; les photographies des lig. 5, 6, 7, 8, 9 proviennent du British Museum; la photographie 11 a éte fournie par le Musée Paul Getty ; les fig. $3 \mathrm{I}, 32,33$ sont de .1. Cl. Bassier ; le dessin de la tig. 30 "st de M. R. Prudhomme. 